Pacific Journal of Mathematics

$C^{*}$-ALGEBRAS OF TRANSFORMATION GROUPS WITH 


\title{
C*-ALGEBRAS OF TRANSFORMATION GROUPS WITH SMOOTH ORBIT SPACE
}

\author{
PhILIP GREeN
}

We investigate the structure of certain locally compact Hausdorff transformation groups $(G, X)$ and the $C^{*}$-algebras $C^{*}(G, X)$ associated to them. When $G$ and $X$ are second countable and the action is free, we obtain a simple necessary and sufficient condition for $C^{*}(G, X)$ to be a continuous trace algebra, and show that the continuous trace algebras so arising are never "twisted" over their spectra. When G is separable compactly generated Abelian and $X$ contains a totally disconnected set of fixed points whose complement, $Z$, is a trivial $G$-principal fiber bundle over its orbit space $Z / G$, with $Z / G$ compact, $C^{*}(G, X)$ can be described completely using the Brown-Douglas-Fillmore theory of extensions of the compact operators on a separable Hilbert space by a commutative algebra. These results yield as special cases the structure of the $C^{*}$-algebras for several infinite families of solvable locally compact groups.

In greater detail the contents of the paper are as follows: In $\S 1$ we discuss, after some preliminary lemmas, $C^{*}(G, X)$ for $X$ having a dense free orbit with complement a finite set of fixed points. (The possible structure of such transformation groups is closely related to the "end theory" of G.) Complete results are obtained when $G$ is separable compactly generated abelian. As an application we compute the group $C^{*}$-algebras of the " $a x+b$ " groups over all nondiscrete locally compact fields. (The real case was first treated by Z'ep [30], the complex case by Rosenberg [22], while the $p$-adic result was found independently by Rosenberg and myself. The methods here are considerably less computational than those of [22] and [30], and in particular we avoid solving any integral equations.)

In $\S 2$ we prove a result which permits us to reduce the study of $C^{*}(G, X)$ in certain cases to the study of algebras of simpler transformation groups. We then apply this to $(G, X)$ for which (1) $G$ is compactly generated, (2) the set $Y$ of fixed points of $X$ is totally disconnected, (3) the complement $Z=X \backslash Y$ of $Y$ is a trivial $G$-principal fiber bundle [24] over $Z / G$, and (4) $Z / G$ is compact, obtaining a complete description of $C^{*}(G, X)$ when $G$ is in addition separable abelian. These results are applied to find the $C^{*}$-algebras of some further locally compact groups, including a family of solvable Lie groups whose $C^{*}$-algebras were first computed in [22]. 
(Again, our methods are simpler than those of [22].)

In the final third section we discuss free actions, i.e., those for which the stabilizer of any point consists of the identity element alone. We show, for second countable $G$ and $X$, that $C^{*}(G, X)$ is a continuous trace algebra if and only if compact subsets of $X$ are "wandering," and that when this happens the corresponding element of $H^{3}(X / G, Z)$ is always 0 . Under suitable smoothness hypotheses it follows that $C^{*}(G, X)$ is isomorphic to the algebra $C_{\infty}(X / G$, $\left.\mathscr{K}\left(L^{2}(G)\right)\right)$ of continuous functions vanishing at infinity on $X / G$ with values in the compact operators on $L^{2}(G)$. We also give an example of a free action of $R$ on a closed subset $X$ of $R^{3}$ for which $X / R$ is Hausdorff but $C^{*}(\boldsymbol{R}, X)$ is not a continuous trace algebra.

We will use the notational conventions introduced above throughout the paper, so that in particular $G$ will always denote a locally compact group, $X$ a locally compact Hausdorff space on which $G$ acts, and $X / G$ the associated orbit space with the quotient topology. Except where noted we work with left Haar measure on $G$, and all actions are jointly continuous left actions. The following shorthand will be used: "group" means "locally compact group": "representation" means "strongly continuous unitary representation" when referring to $G$ and "nondegenerate *-representation" when referring to a $C^{*}$-algebra; "ideal" means "closed two-sided ideal." We use $\boldsymbol{Z}$ and $\boldsymbol{R}$ to denote the integers and real numbers, respectively.

The basic facts about $C^{*}$-algebras, as covered in the first four chapters of [8], will be used without special reference.

I would like to thank my adviser, Professor Marc Rieffel, for a number of helpful suggestions which eliminated obscurities from the exposition.

1. Transformation groups with finitely many orbits. We refer the reader to [13] or [10] for the definition of $C^{*}(G, X)$ and for the proof of the following fundamental fact: representations $L$ of $C^{*}(G, X)$ on a Hilbert space $\mathscr{H}$ are in natural one-to-one correspondence with pairs $(V, M)$ where $V=V_{L}$ is a representation of $G$ on $\mathscr{H}$, and $M=M_{L}$ a representation of $C_{\infty}(X)$ on $\mathscr{H}$, such that $V(s) M(f) V\left(s^{-1}\right)=M(s f)$ for every $s$ in $G$ and $f$ in $C_{\infty}(X)$. (Here $s f(x)=f\left(s^{-1} x\right)$ for all $x$ in $X$.) We will also need the fact, implicit in [10], that there are natural homomorphisms (denoted $\varphi_{G}$ and $\varphi_{X}$ respectively) of $C^{*}(G)$ and $C_{\infty}(X)$ into the multiplier algebra (cf. [1]) of $C^{*}(G, X)$ such that for any representation $L$ of $C^{*}(G, X), V_{L}=$ $L \circ \varphi_{G}$ and $M_{L}=L \circ \varphi_{X}$ (Here and in the following we use the same letter for the associated representations of a group and its $C^{*}$ algebra and similarly for the associated representations of a $C^{*}$ algebra and its multiplier algebra.) For any $k$ in $C^{*}(G)$ and $f$ in 
$C_{\infty}(X)$ the product $\varphi_{G}(k) \cdot \varphi_{X}(f)$ is in $C^{*}(G, X)$; we will denote it by $k \cdot f$ (and similarly will let $f \cdot k$ denote $\left.\varphi_{X}(f) \cdot \varphi_{G}(k)\right)$. The set of all such products generates $C^{*}(G, X)$ as a $C^{*}$-algebra.

The first lemma below summarizes some basic facts about the ideal structure of $C^{*}(G, X)$. Before stating it we recall that if $Z$ is an open subset of $X, C_{\infty}(Z)$ can be identified with the ideal of $C_{\infty}(X)$ consisting of functions which vanish off $Z$. We will frequently make this identification, but when it is necessary to distinguish between $f \in C_{\infty}(Z)$ and the corresponding element of $C_{\infty}(X)$ we will use $f^{x}$ for the latter. Part (i) of the following lemma will enable us to identify $C^{*}(G, Z)$ with an ideal of $C^{*}(G, X)$ when $Z$ is a $G$ invariant; again, this identification will frequently be made when no confusion is likely to result.

Lemma 1. Let $Z$ be an open G-invariant subset of $X$.

(i) There is an isomorphism $\alpha_{Z}$ of $C^{*}(G, Z)$ onto an ideal of $C^{*}(G, X)$ such that, for $k$ in $C^{*}(G)$ and $f$ in $C_{\infty}(Z), \alpha_{Z}(k \cdot f)=k \cdot f^{X}$. The map so induced from open $G$-invariant subsets of $X$ to ideals of $C^{*}(G, X)$ is one-to-one and preserves finite intersections and arbitrary unions (the 'union' of a collection of ideals being the ideal they generate).

(ii) The kernel $\operatorname{Ker} L$ of a representation $L$ of $C^{*}(G, X)$ contains $C^{*}(G, Z)$ if and only if $\operatorname{Ker} M_{L} \supseteqq C_{\infty}(Z)$. If $L$ is irreducible then hull $\left(\operatorname{Ker} M_{L}\right)$ is not the union of two proper closed G-invariant subsets of $X$. (For any representation $L$, hull $\left(\operatorname{Ker} M_{L}\right)$ is closed and $G$-invariant, by [10, p. 62].)

(iii) Let $Y=X \backslash Z$. There is a surjective homomorphism $\beta_{Y}$ : $C^{*}(G, X) \rightarrow C^{*}(G, Y)$ with kernel $C^{*}(G, Z)$, such that $\beta_{Y}(k \cdot f)=\left.k \cdot f\right|_{Y}$ for $k$ in $C^{*}(G)$, $f$ in $C_{\infty}(X)$.

Proof. Choose a faithful representation $L$ of $C^{*}(G, X)$ and let $V=V_{L}, M=M_{L}$. Then $V$ and $\left.M\right|_{c_{\infty}(Z)}$ define a (possibly degenerate) representation $L_{0}$ of $C^{*}(G, Z)$. Since $L_{0}\left(C^{*}(G, Z)\right)$ is generated by the subset $\left\{V(k) M(f): k \in C^{*}(G), f \in C_{\infty}(Z)\right\}$, which is mapped into $L_{0}\left(C^{*}(G, Z)\right)$ by left or right multiplication by anything of the form $V(h)$ or $M(g)$ (with $h$ in $C^{*}(G)$ and $g$ in $C_{\infty}(X)$ ), it is clear that the image of $C^{*}(G, Z)$ is an ideal of $L\left(C^{*}(G, X)\right)$. Taking $\alpha_{z}=L^{-1} \circ L_{0}$, we see $\alpha_{z}(k \cdot f)=k \cdot f^{X}$ for $k$ in $C^{*}(G)$ and $f$ in $C_{\infty}(Z)$. Furthermore $\alpha_{Z}$ must be injective since any representation $K$ of $C^{*}(G, Z)$ "extends" to a representation $H$ of $C^{*}(G, X)$ with $V_{H}$ equal $V_{K}$ and $M_{H}$ equal to the canonical extension of $M_{K}$ to $C_{\infty}(X)$.

If $L$ is now any representation of $C^{*}(G, X)$ then $\operatorname{Ker} L \supseteqq C^{*}(G, Z)$ iff $\operatorname{Ker} L \supseteqq\left[k \cdot f: k \in C^{*}(G), f \in C_{\infty}(Z)\right\}$, iff $V_{L}(k) \cdot M_{L}(f)=0$ for all $k$ in $C^{*}(G)$ and $f$ in $C_{\infty}(Z)$; but since $V_{L}$ is nondegenerate the latter 
can happen only if $M_{L}$ annihilates $C_{\infty}(Z)$. Now let $Z_{1}$ and $Z_{2}$ be open $G$-invariant subsets of $X$, with $Z_{1} \nsubseteq Z_{2}$, and choose $z$ in $Z_{1} \backslash Z_{2}$. Letting $H$ be the stabilizer in $G$ of $z$ we may project a quasi-invariant measure on $G / H$ to get a measure $\mu$ on the orbit $E=G z$, and then construct a representation $L$ of $C^{*}(G, X)$ on $L^{2}(E, \mu)$ in the obvious way- $G$ acts by left translation (modified by Radon-Nikodym derivatives so as to give a unitary representation), while $C_{\infty}(X)$ acts by multiplication of functions. Then $L$ annihilates $C^{*}\left(G, Z_{2}\right)$ but not $C^{*}\left(G, Z_{1}\right)$, so the indicated map from invariant open sets to ideals is indeed one-to-one. The intersection of $C^{*}\left(G, Z_{1}\right)$ and $C^{*}\left(G, Z_{2}\right)$ clearly contains $C^{*}\left(G, Z_{1} \cap Z_{2}\right)$, for arbitrary open invariant $Z_{1}$ and $Z_{2}$; the reverse inclusion follows from the fact that for $k_{i} \in C^{*}(G)$ and $f_{i} \in C_{\infty}\left(Z_{i}\right)(i=1,2)$ we have $k_{1} \cdot f_{1} \cdot f_{2} \cdot k_{2} \in C^{*}\left(G, Z_{1} \cap Z_{2}\right)$ (and the fact that the intersection of two ideals is equal to their product). For a family $\left\{Z_{j}\right\}$ of open invariant sets obviously $\cup_{j} C^{*}\left(G, Z_{j}\right) \subseteq C^{*}(G, W)$ where $W=\cup_{j} Z_{j}$; since $C_{c}(W)$ (the algebra of complex valued functions with compact support) is dense in $C_{\infty}(W)$, and since any element of $C_{c}(W)$ can be written as a finite sum $\sum_{i=1}^{n} f_{i}$, with each $f_{i}$ in $C_{o}\left(Z_{j(i)}\right)$ for some $j(i)$, we have that $\cup_{j}\left(C^{*}(G) \cdot C_{\infty}\left(Z_{j}\right)\right)$ generates $C^{*}(G, W)$.

At this point we have proved (i) and the first statement of (ii). The second statement of (ii) follows from the fact that the kernel of an irreducible representation of a $C^{*}$-algebra is a prime ideal, so that if $\operatorname{Ker} L \nsupseteq C^{*}\left(G, Z_{i}\right)$ for $i=1,2$ then $\operatorname{Ker} L \nsupseteq C^{*}\left(G, Z_{1}\right) \cap C^{*}(G$, $Z)=C^{*}\left(G, Z_{1} \cap Z_{2}\right)$. To prove part (iii) observe that a representation $K$ of $C^{*}(G, Y)$ defines a representation $H$ of $C^{*}(G, X)$ (take $M_{H}$ to be the composition of $M_{K}$ with the natural homomorphism of $C_{\infty}(X)$ onto $C_{\infty}(Y)$ ), and that by (ii) a representation of $C^{*}(G, X)$ factors through $C^{*}(G, Y)$ if and only if it annihilates $C^{*}(G, Z)$.

We will see later that for many interesting transformation groups $(G, X)$ it is possible to find an open $G$-invariant subset $Z$ such that $C^{*}(G, Z)$ and $C^{*}(G, Y)(Y=X \backslash Z)$ are known; by the above lemma determination of $C^{*}(G, X)$ then reduces to describing how $C^{*}(G, Y)$ "extends" $C^{*}(G, Z)$. The general problem of computing the extensions of one $C^{*}$-algebra by another is very difficult, but for certain algebras significant progress has been made in recent work of Brown, Douglas, and Fillmore ("BDF"). (See [2], [3].) In the following lemma we adapt their results slightly to provide a framework for describing the extensions encountered later in this section. (For unexplained terminology we refer the reader to [2].) $\mathscr{L}(\mathscr{H})$ and $\mathscr{K}(\mathscr{H})$ will denote respectively the algebras of all bounded operators and all compact operators on the Hilbert space $\mathscr{H}$. 
Lemma 2. Let $A$ be a separable commutative $C^{*}$-algebra, and $\mathscr{H}$ a separable infinite dimensional Hilbert space. (i) Weak equivalence classes of extensions of $\mathscr{K}(\mathscr{H})$ by $A$ are in one-to-one correspondence with the set $T_{A}$ of triples $(X, C, \gamma)$ where $X$ is the spectrum of $A, C$ is a closed subset of the one point compactification $X^{\sim}$ of $X$, and $\gamma$ is an element of the BDF abelian group $\operatorname{Ext}(C)$.

(ii) Let $A$ be the $C^{*}$-algebra direct sum of a countable family $\left\{A_{i}: i \in \mathscr{F}\right\}$ of separable commutative $C^{*}$-algebras. Then any extension of $\mathscr{K}(\mathscr{H})$ by $A$ is isomorphic to a "direct sum" of extensions of $\mathscr{K}(\mathscr{H})$ by $A_{i}$ for $i \in \mathscr{F}$; this decomposition implements a natural embedding of $T_{A}$ in $\prod_{i \in \mathcal{S}} T_{A_{i}}$.

Proof. (i) Weak equivalence classes of extensions are in 1-1 correspondence with equivalence classes of homomorphisms of $A$ into the Calkin algebra $\mathscr{L}(\mathscr{H}) / \mathscr{K}(\mathscr{H})$, two such homomorphisms being equivalent if they are "intertwined" by a unitary operator in $\mathscr{L}(\mathscr{C})$. (This result is essentially due to Busby [4], although he used a different definition of weak equivalence; we are using that of [2].) When $A$ has a unit and the homomorphisms are faithful and unit preserving their equivalence classes are parameterized by $\operatorname{Ext}(X)$; the general case follows from this since an arbitrary homomorphism determines a faithful unit preserving homomorphism of a quotient algebra of $A^{\sim}$ (the algebra formed by adjoining a unit to $A$ ).

(ii) When there are only finitely many nonzero $A_{i}$ this is a consequence of [2, 4.8]. For infinitely many nonzero $A_{i}$, observe that $X^{\sim}$ can be identified with the "strong wedge" of the $X_{i}^{\sim}([15])$. For any closed subset $C$ of $X^{\sim}$, either $C$ contains the point at infinity, in which case $C$ is the strong wedge of the sets $C_{i}=C \cap X_{i}$, or else $C_{i}$ is empty for all but finitely many $i$. The desired result can now be easily deduced from [2, 8.2].

We now proceed to show that certain transformation groups give rise to algebras describable by the $\mathrm{BDF}$ theory. The (wellknown) lemma below is a first step in that direction. Here $(G, G)$ is the transformation group obtained by letting $G$ act on itself by left translation.

Lemma 3. Let $L$ be the representation of $C^{*}(G, G)$ on $L^{2}(G)$ such that $V_{L}$ is the left regular representation of $G$, and $M_{L}$ represents $C_{\infty}(G)$ by multiplication of functions. Then $L$ is faithful and identifies $C^{*}(G, G)$ with $\mathscr{K}\left(L^{2}(G)\right)$. 
Proof. See [25, Prop. 3.3] or (for a more elegant proof) [20].

In the remainder of this section we consider $(G, X)$ such that $X$ contains an open invariant subset $Z$ with $(G, Z)$ isomorphic to $(G, G)$ and with $Y=X \backslash Z$ a finite collection of $G$-fixed points. Before proceeding further it is worth pointing out that there is a close connection, made precise in the following lemma (which will not actually be needed until the next section), between transformation groups of this type and the theory of ends of compactly generated groups. (For the definitions and basic facts of this theory used in the following, see [20, pp. 18-21].) For any compactly generated group $G$ the disjoint union $E=G \cup \xi$, where $\xi$ is the set of ends of $G$, carries a natural compact Hausdorff topology such that, with $G$ acting on itself by left translation and fixing $\xi$ pointwise, $(G, E)$ is a transformation group. With this notation we have:

LEMMA 4. Let $G$ be compactly generated, and suppose $X$ is compact and contains a totally disconnected set $Y$ of G-fixed points such that $X \backslash Y=G$ (as a transformation group) and is dense in $X$. Then there is a unique G-equivariant continuous map $\varphi$ of $E$ onto $X$ preserving the subset $G$ (of $E$ and $X$ ).

Proof. As $Y$ is compact (since, being the set of fixed points, it is closed in $X$ ) and totally disconnected, any $y$ in $Y$ has a basis $\mathscr{N}_{y}$ for its neighborhoods (in $X$ ) which consists of compact sets $Q$ such that $Q \cap Y$ is open in $Y$. Let $\mathscr{C}_{y}$ denote the family of subsets of $G$ of the form $G \cap Q$ for $Q$ in $\mathscr{N}_{y}$.

Observe that each end $\varepsilon$ of $G$ contains $\mathscr{C}_{z}$ for some $z$ in $Y$ : Otherwise by compactness of $Y$ we could find a finite family $\left\{Q_{i}\right\}$ of subsets of $X$ with union $Q$ a neighborhood of $Y$, and with each $Q_{i}$ in $\mathscr{N}_{y_{i}}$ for some $y_{i}$, but with $Q_{i} \cap G$ not in $\varepsilon$. Now (in the terminology of [20, pp. 19-20]) $Q_{i} \cap G$ is "unbounded" in $G$ (otherwise $y_{i}$ could not be in the closure of $G$ ); and given any "seed" $U$ of $G$, since $Y$ is fixed by $U$ we have

$$
\left(U \cdot\left(P_{i} \cap G\right)\right) \cap\left(U \cdot\left(Q_{i} \cap G\right)\right)=U P_{i} \cap U Q_{i}
$$

where $P_{i}$ is the complement of $Q_{i}$ in $X$. Since $U P_{i} \cap U Q_{i}$ is relatively compact in $G$ we see that $Q_{i} \cap G$ is a "neighborhood of infinity" in $G$. Then from the maximality condition for ends [20, p. 20], $Q \cap G=\cup_{i}\left(Q_{i} \cap G\right)$ cannot be in $\varepsilon$ (otherwise one of the $Q_{i} \cap G$ would have to be); but this contradicts the fact that $Q \cap G$, as a set with bounded complement in $G$, must be contained in every end ([20, p. 20]).

Thus $\varepsilon$ contains some $\mathscr{M}_{z}$. As $X$ is Hausdorff and any two 
elements of $\varepsilon$ must intersect (as subsets of $G$ ), $\varepsilon$ can contain only one $\mathscr{C}_{z}$. Hence we get a natural map $\xi \rightarrow Y$, which extends (by leaving each $s$ in $G$ fixed) to a map $\varnothing$ from $E$ to $X$. It is then easy to see (using the definition of the topology on $E$ ) that $\varphi$ is continuous, and hence, by density of $G$ in $X$ and compactness of $E$, surjective; $\varphi$ is $G$-equivariant by construction.

Uniqueness follows from the density of $G$ in $E$.

Given an $X$ as in the lemma but not compact, $X \sim$ (with the obvious $G$ action) satisfies all the conditions of the lemma. Hence in either case the number of fixed points of $X$ is less than or equal to the number of ends of $G$. It is known ([20,4.3]) that a compactly generated group has either $0,1,2$ or infinitely many ends. Seemingly the great majority of groups have exactly one end; the groups with two ends are precisely those having a discrete uniform subgroup isomorphic to $\boldsymbol{Z}$, while the finitely generated discrete groups with infinitely many ends are, roughly speaking, always free products (see [6] for a more precise statement of this result, which is due to Stallings). Among connected groups only those of the from $\boldsymbol{R} \times K$ with $K$ compact have two ends (a result apparently due to Freudenthal-cf. [20, p. 10]), all others having one end (except for the compact groups, which have no ends). A noncompact compactly generated abelian group always has one end unless it is of the form $Z \times K$ or $\boldsymbol{R} \times K$ (in which case it has 2 ends).

We turn now to the task of describing the $C^{*}$-algebras of transformation groups of the type just discussed. Let $(G, X)$ be as in the preceding lemma, with the additional restriction that $Y$ be finite, but with $X$ not assumed to be compact. Since $C^{*}(G$, point $) \cong$ $C^{*}(G)$, we see easily from Lemma 1 that $C^{*}(G, Y)$ is a finite direct sum of copies of $C^{*}(G)$. When $G$ is separable abelian we may, in view of Lemmas 1 and 3 , describe $C^{*}(G, X)$ by producing a triple of the sort described in Lemma 2. Note that, since we are interested in describing only the isomorphism class of $C^{*}(G, X)$, there is some ambiguity in the choice of $\gamma(\mathrm{cf}$. the discussion on pp. 120-121 of [2]). In particular for the algebras arising in the next few lemmas the corresponding Ext group is a direct product of copies of $\boldsymbol{Z}$, and it is easy to see that different $\gamma$ 's in this group will define the same algebra if the absolute values of their components agree; we choose $\gamma$ 's with nonnegative components, by convention.

LEMMA 5. Let $X$ be the two point compactification $\boldsymbol{Z} \cup\{ \pm \infty\}$ of $\boldsymbol{Z}$, and let $G=\boldsymbol{Z}$ act on $X$ by fixing the points at infinity and "left translating" the remainder. Then $C^{*}(G, X)$ is isomorphic to 
an abelian extension of $\mathscr{K}\left(l^{2}(\boldsymbol{Z})\right)$ with triple $\left(\boldsymbol{T} \times \boldsymbol{Z}_{2}, \boldsymbol{T} \times \boldsymbol{Z}_{2},(1,1)\right)$, where $\boldsymbol{T}$ denotes the unit circle, $\boldsymbol{Z}_{2}=\boldsymbol{Z} / 2 \boldsymbol{Z}$, and $(1,1) \in \boldsymbol{Z} \oplus \boldsymbol{Z} \cong$ $\operatorname{Ext}\left(\boldsymbol{T} \times \boldsymbol{Z}_{2}\right)$. Let $X_{1}=X \backslash\{-\infty\}$; then $C^{*}\left(G, X_{1}\right)$ has triple $(\boldsymbol{T}, \boldsymbol{T} \cup$ $\{0\}, 1)$, where $1 \in Z \simeq \operatorname{Ext}(\boldsymbol{T} \cup\{0\})$.

Proof. Let $L$ be the representation of $C^{*}(G, X)$ on $l^{2}(Z)$ such that $V_{L}$ is the left regular representation of $Z$ and $C_{\infty}(X)$ acts by multiplication of functions. By Lemma $3, L\left(C^{*}(\boldsymbol{Z}, \boldsymbol{Z})\right)=\mathscr{K}\left(l^{2}(\boldsymbol{Z})\right)$. Now define $f$ in $C_{\infty}\left(X_{1}\right)$ by $f(r)=1$ for $r \geqq 0$ and $f(r)=0$ for $r<0$, and let $k_{i} \in C_{c}(\boldsymbol{Z})$ (regarded as a subalgebra of $C^{*}(\boldsymbol{Z})$ ) be the characteristic function of the singleton $\{i\}$, for each $i$ in $Z$. Then the operator

$$
T=1_{l}^{2}-V_{L}\left(k_{0}\right) \cdot M_{L}(f)+V_{L}\left(k_{1}\right) \cdot M_{L}(f)
$$

acts as the unilateral shift on $l^{2}([0, \infty))$ and as the identity operator on $l^{2}((-\infty,-1])$, where those two spaces are regarded as subspaces of $l^{2}(\boldsymbol{Z})$ in the natural manner. The adjoint of $T$ is therefore a Fredholm operator with index 1.

We have shown that $L\left(C^{*}\left(G, X_{1}\right)^{\sim}\right)$ contains an operator with non-trivial Fredholm index, so by [2, Thm. 10.5] the algebra $A=$ $L\left(C^{*}\left(G, X_{1}\right)^{\sim}\right) / \mathscr{K}\left(l^{2}(\boldsymbol{Z})\right)$ cannot have its spectrum $A^{\wedge}$ embeddable in $\boldsymbol{R}$. Since, by Lemma 1 (iii) and the fact that $L$ identifies $C^{*}(Z, Z)$ with $\mathscr{K}\left(l^{2}(\boldsymbol{Z})\right), A$ is a quotient algebra of $C^{*}(G \text {, point })^{\sim}$, and so has spectrum isomorphic to a closed subset of $\boldsymbol{T}^{\sim} \cong \boldsymbol{T} \cup\{0\}$, either $A^{\wedge} \cong$ $\boldsymbol{T}$ or $A^{\wedge} \cong \boldsymbol{T} \cup\{0\}$; but the first possibility is ruled out by the fact that the operator $U=V_{L}\left(k_{1}\right) M_{L}(f)$, being the direct sum of a unilateral shift and a zero operator, has essential spectrum $\boldsymbol{T} \cup\{0\}$, which is not a continuous image of $T$. It is now clear that $C^{*}(G$, $X_{1}$ ) has the invariants claimed in the lemma, since extensions with $\gamma \in \operatorname{Ext}(\boldsymbol{T} \cup\{0\})$ equal to 0 , or with $|\gamma| \geqq 2$, can contain no operators with Fredholm index 1 (this may be seen from [2, Thm. 10.5] for example). The invariants for $C^{*}(G, X)$ now follow from the fact that they are determined by the values "at each piece" of the spectrum of $C^{*}(\boldsymbol{Z},\{ \pm \infty\})$, as in Lemma 2 .

Lemma 6. Let $G=\boldsymbol{R}$ and $X=[-\infty, \infty]$, and let $G$ act on $X$ by translation (fixing $\pm \infty)$. Then $C^{*}(G, X)$ has triple $\left(\boldsymbol{R} \times \boldsymbol{Z}_{2}, S\right.$, $(1,1)$ ) where $S$ is the one point compactification of $\boldsymbol{R} \times \boldsymbol{Z}_{2}$ and $(1,1) \in Z \oplus \boldsymbol{Z} \cong \operatorname{Ext}(S)$. Let $X_{1}=X \backslash\{-\infty\}$, and identify $\boldsymbol{R}^{\sim}$ with $\boldsymbol{T}$; then $C^{*}\left(G, X_{1}\right)$ has triple $(\boldsymbol{R}, \boldsymbol{T}, 1)$.

Proof. Since the argument is practically identical in form to that of the preceding lemma we concentrate on the main difference, which is the construction of an operator of Fredholm index 1 . We 
regard $L^{2}(\boldsymbol{R})$ as the direct sum of $\mathscr{H}_{-}=L^{2}((-\infty, 0))$ and $\mathscr{H}_{+}=$ $L^{2}((0, \infty))$, and produce an operator which acts as the identity on $\mathscr{H}_{-}$, and on $\mathscr{H}_{+}$as a unilateral shift with respect to the orthonormal basis $\left\{L_{n}(t) e^{-t / 2}: n=0,1,2 \cdots\right\}$ where $L_{n}$ is the $n^{-t h}$ Laguerre polynomial: $L_{n}(t)=\sum_{i=0}^{n}\left(\begin{array}{l}n \\ i\end{array}\right)(-t)^{j} / i !$. (For the basic facts about the Laguerre polynomials, consult [25, Chapter 5].) First we observe that the operator $T=V_{L}(k) M_{L}(f)$-where $f$ is the characteristic function of $[0, \infty), k=e^{-t / 2} f$, and $L$ is constructed as in the preceding lemma-is in $L\left(C^{*}\left(G, X_{1}\right)\right)$, even though $f$ is not in $C_{\infty}\left(X_{1}\right)$ : choose $f_{j}$ in $C_{\infty}\left(X_{1}\right)$ for $j=1,2, \cdots$ such that $f_{j}(x)=1$ for $x \geqq 0, f_{j}(x)=0$ for $x \leqq-1 / j$, and $\left|f_{j}(x)\right| \leqq 1$ for all $x$. Then for any $h$ in $L^{2}(R)$ a straight-forward calculation shows that

$$
\left|V(k) M\left(f_{j}-f\right)(h)(x)\right| \leqq e^{-x / 2} \chi_{[-1 / j, \infty)}(x) \mid\left(\int_{-1 / j}^{0}|h(t)| d t\right)
$$

for all $x$ in $R$ (where $\chi_{[-1 / j, \infty)}$ is the characteristic function of $[-1 / j$, $\infty)$ ), and so

$$
\begin{aligned}
\left\|V(k) M\left(f_{j}-f\right)(h)\right\|_{2} & \leqq\left\|e^{-x / 2} \chi_{[-1 / j, \infty)}\right\|_{2} j^{-1 / 2}\|h\|_{2} \\
& =(\exp (1 / 2 j)) j^{-1 / 2}\|h\|_{2} .
\end{aligned}
$$

It follows that $T$ is the norm limit of the operators $V(k) M\left(f_{j}\right)$, and is thus in $L\left(C^{*}\left(G, X_{1}\right)\right)$. The operator $I_{L^{2}(R)}-T$ acts as the identity on $L^{2}((-\infty, 0))$ while for $h \in L^{2}((0, \infty))$ we have

$$
((I-T) h)(x)=h(x)-e^{-x / 2} \int_{0}^{x} e^{t / 2} h(t) d t .
$$

Using the above explicit formula for $L_{n}(t)$ we see easily that

$$
\begin{aligned}
& (I-T)\left(L_{n}(x) e^{-x / 2}\right)=e^{-x / 2}\left(L_{n}(x)-\int_{0}^{x} L_{n}(t) d t\right) \\
& \quad=e^{-x / 2}\left(\sum_{i=0}^{n}\left(\begin{array}{c}
n \\
i
\end{array}\right)(-x)^{i} / i !+\sum_{i=1}^{n+1}\left(\begin{array}{c}
n \\
i-1
\end{array}\right)(-x)^{i} / i !\right)=L_{n+1}(x) e^{-x / 2}
\end{aligned}
$$

so $I-T$ is indeed a shift with respect to this basis for $\mathscr{H}_{+}$. The adjoint of $I-T$ is then an operator in $L\left(C^{*}\left(G, X_{1}\right)^{\sim}\right)$ with Fredholm index 1. The rest of the argument proceeds as in Lemma 5.

Proposition 7. In the following $K$ is any separable compact abelian group. (i) Let $G=K \times Z$, and let $X$ be the two point compactification $(K \times \boldsymbol{Z}) \cup\{ \pm \infty\}$ of $G$, with $G$ acting on $X$ by fixing the points at infinity and left translating the remainder. Then $C^{*}(G, X)$ is an abelian extension of (an algebra isomorphic to) $\mathscr{K}\left(L^{2}(G)\right)$, with extension triple $\left(T \times K^{\wedge} \times Z_{2}, S,((1,1, \cdots),(1,1\right.$, $\cdots))$ ), where $S$ is $\boldsymbol{T} \times K^{\wedge} \times \boldsymbol{Z}_{2}$ if $K$ is finite, and $\left(\boldsymbol{T} \times K^{\wedge} \times \boldsymbol{Z}_{2}\right)^{\sim}$ 
otherwise, and $((1,1, \cdots),(1,1, \cdots)) \in\left(\prod_{x \in K^{\wedge}} \boldsymbol{Z}\right) \times\left(\prod_{x \in K^{\wedge}} \boldsymbol{Z}\right) \cong \operatorname{Ext}(S)$. Let $X_{1}=X \backslash\{-\infty\}$; then $C^{*}\left(G, X_{1}\right)$ has triple $\left(\boldsymbol{T} \times K^{\wedge}, S,(1,1, \cdots)\right)$ where $S=\left(\boldsymbol{T} \times K^{\wedge}\right)^{\sim}$.

(ii) Let $G=K \times \boldsymbol{R}$, and let $X$ be the two point compactification $(K \times \boldsymbol{R}) \cup\{ \pm \infty\}$ of $G$. ( $X$ is homeomorphic to the unreduced suspension of $K$.) Then with $G$ acting on $X$ as in (i), $C^{*}(G, X)$ has triple $\left(\boldsymbol{R} \times K^{\wedge} \times \boldsymbol{Z}_{2},\left(\boldsymbol{R} \times K^{\wedge} \times \boldsymbol{Z}_{2}\right)^{\sim},((1,1, \cdots),(1,1, \cdots))\right)$. Let $X_{1}=X \backslash\{-\infty\}$; then $C^{*}\left(G, X_{1}\right)$ has triple $\left(\boldsymbol{R} \times K^{\wedge},\left(\boldsymbol{R} \times K^{\wedge}\right)^{\sim}\right.$, $(1,1, \cdots))$.

Proof. We prove (i) only, the argument for (ii) being virtually identical. As in the two preceding lemmas we represent $C^{*}\left(G, X_{1}\right)$ on $L^{2}(G)$. By Lemma $1 C^{*}\left(G, X_{1}\right)$ is an extension of $C^{*}(G, G)$ by $C^{*}(G,\{\infty\}) \cong C^{*}(G)$. Now $C^{*}(G) \cong C_{\infty}\left(\boldsymbol{T} \times K^{\wedge}\right)$ is the $C^{*}$-algebra direct sum of the subalgebras $C_{\chi}^{*}(\boldsymbol{Z}) \cong C_{\infty}(\boldsymbol{T} \times\{\chi\})\left(\chi \in K^{\wedge}\right)$, hence in view of Lemma 2(ii) we can describe the extension by decomposing it into a "direct sum" of extensions by the $C_{x}^{*}(\boldsymbol{Z})$. This is done as follows:

Using the Plancherel theorem for $K$ we may write $L^{2}(G) \cong$ $\bigoplus_{\chi \in K^{\wedge}} l_{\chi}^{2}(\boldsymbol{Z})$, with $l_{\chi}^{2}(\boldsymbol{Z}) \cong l^{2}(\boldsymbol{Z})$ for each $\chi$. Given a fixed $\chi_{1} \in K^{\wedge}$ it is easily checked that $C_{\chi_{1}}^{*}(\boldsymbol{Z}) \cong C^{*}(\boldsymbol{Z})$ acts on $\ell_{\chi_{1}}^{2}(\boldsymbol{Z})$ as in the regular representation of $Z$, while it annihilates $\ell_{\chi}^{2}(Z)$ for $\chi \neq \chi_{1}$. Furthermore, if we denote by $A$ the subalgebra of $C_{\infty}\left(X_{1}\right)$ consisting of functions whose restrictions to $K \times Z$ are constant on cosets of $K$, then any $f$ in $A$ acts on $\ell_{x_{1}}^{2}(Z)$ as multiplication by the bounded function $f_{*}$ on $Z$, where $f_{*}(r)=f(0, r)$ for each $r$ in $Z$. It follows easily from Lemma 5 that the $C^{*}$-algebra on $\ell_{\chi_{1}}^{2}(\boldsymbol{Z})$ generated by (the restrictions of) $V\left(C_{\chi_{1}}^{*}(\boldsymbol{Z})\right) M(A)$ is an extension of $\mathscr{K}\left(\ell_{\chi_{1}}^{2}(\boldsymbol{Z})\right)$ with triple $(\boldsymbol{T}, \boldsymbol{T} \cup\{0\}, 1)$.

But now $C_{\infty}\left(X_{1}\right)$ is the linear span of $A$ and the subalgebra $B$ of functions which vanish at $\infty$, so $L\left(C^{*}\left(G, X_{1}\right)\right)$ is generated by the above algebras on the $\ell_{x}^{2}(Z)$ together with $V\left(C^{*}(G)\right) M(B)$. By Lemma 3 the latter algebra is just $\mathscr{K}\left(L^{2}(G)\right)$. We can now readily deduce from the definition [2, 8.1] of "direct sum" extensions that $C^{*}\left(G, X_{1}\right)$ has the form claimed in the Proposition.

A similar argument gives the structure of $C^{*}(G, X)$.

From the above proposition we can easily deduce the structure of $C^{*}(H)$ for $H$ the " $a x+b$ " group over an arbitrary nondiscrete locally compact field $F$. (For the facts about such fields used below, the reader may consult Chapter 2 of [5].) This group is defined to be the semidirect product of the underlying additive group $F^{+}$of $F$ with the multiplicative group $F^{*}$ of nonzero elements 
of $F$, acting on $F^{+}$by multiplication. It is well known (and is easy to deduce from the basic facts about transformation group algebras quoted at the beginning of this section) that the group $C^{*}$-algebra of the semidirect product of a locally compact group $G$ acting on an abelian locally compact group $A$ is just $C^{*}\left(G, A^{\wedge}\right)$, where $A^{\wedge}$ is given the dual action of $G$. In the case of the " $a x+b$ " group, $G=F^{*}$ and the dual action on $\left(F^{+}\right)^{\wedge} \cong F^{+}$is (isomorphic to) ordinary multiplication. We have $F^{*} \cong \boldsymbol{Z} \times K$ or $F^{*} \cong \boldsymbol{R} \times K$ according as $F$ is non-archimedean or archimedean, where in each case $K$ is the compact group of elements of absolute value 1 , and the summand $\boldsymbol{Z}$ or $\boldsymbol{R}$ is a cross-section for the logarithm of the absolute value map. It is then easy to see that the corresponding transformation group $\left(F^{*},\left(F^{+}\right)^{\wedge}\right)$ is isomorphic to $\left(G, X_{1}\right)$ of the preceding proposition (with $G=F^{*}$; the isomorphism of $\left(F^{+}\right)^{\wedge}$ with $X_{1}$ sends 0 to $\infty)$, and hence that $C^{*}(H)$ has the structure indicated in the proposition for $C^{*}\left(G, X_{1}\right)$.

The transformation groups of Proposition 7 are rather special in that the groups $G$ considered there all have two ends. For groups with one end, the only possible transformation groups of the sort we have been considering are $(G, G)$ and $\left(G, G^{\sim}\right.$ ) (where $G$ acts on $G^{\sim}$ by fixing the point at infinity). In the last result of this section we partially characterize the corresponding $C^{*}$-algebras.

Proposition 8. Let $G$ be arbitrary, and let $X=G^{\sim}$. Then $C^{*}(G, X)$ is a split extension of (an algebra isomorphic to) $\mathscr{K}\left(L^{2}(G)\right)$ by (an algebra isomorphic to) $C^{*}(G)$. If $G$ is amenable and noncompact the extension is essential (i.e., every nontrivial ideal of $C^{*}(G, X)$ contains $\left.C^{*}(G, G)\right)$.

Proof. The map $k \rightarrow k \cdot 1$ from $C^{*}(G)$ to $C^{*}(G, X)$ is easily seen to define a splitting homomorphism for the extension. Therefore we need only check that for amenable noncompact $G$ the extension is essential. Represent $C^{*}(G, X)$ on $\mathscr{H}=L^{2}(G)$ in the usual way (again calling the representation $L$ ) and observe that, since any sub$C^{*}$-algebra of $\mathscr{K}(\mathscr{H})$ containing $\mathscr{K}(\mathscr{H})$ has $\mathscr{K}(\mathscr{H})$ as its unique minimal ideal, it is sufficient to verify that for $G$ as above the representation is faithful. This in turn will follow if we can show that the induced homomorphism

$$
C^{*}(G, X) / C^{*}(G, G) \longrightarrow \mathscr{L}(\mathscr{H}) / \mathscr{K}(\mathscr{H})
$$

is one-to-one, or equivalently that the representation of $C^{*}(G)$ obtained by composing the above splitting map with the representation $L$ of $C^{*}(G, X)$ is faithful and has image intersecting the com- 
pact operators only in (0). Since this representation of $C^{*}(G)$ is just the left regular representation $\lambda$, faithfulness holds precisely when $G$ is amenable. We conclude the proof by showing that for arbitrary noncompact $G$ and arbitrary $k$ in $C^{*}(G)$ the operator $\lambda(k)$ is not compact unless it is 0 . We may assume $\|\lambda(k)\|=1$. Choose $g, h$ in $C_{c}(G)$ so that $\|\lambda(k-h)\|<1 / 4,\|g\|_{2}=1$, and $\|\lambda(k)(g)\|_{2}>$ 3/4. Letting $C$ and $D$ be the respective supports of $h$ and $g$, then since $G$ is noncompact we may find a countable family $\left\{s_{i}, i=1,2\right.$, ... of elements of $G$ such that the subsets $C D s_{i}$ are pairwise disjoint. Define $g_{i} \in C_{c}(G)$ by $g_{i}(x)=g\left(x s_{i}^{-1}\right) \Delta_{G}\left(s_{i}^{-1}\right)^{1 / 2}$ Then $\left\|g_{i}\right\|_{2}=1$ and $\left\|\lambda(h)\left(g_{i}\right)\right\|_{2}=\|\lambda(h) g\|_{2}>1 / 2$. Furthermore since supp $\lambda(h) g_{i} \leqq$ $C D s_{i}$ the $\lambda(h) g_{i}$ are mutually orthogonal, and so $\left\|\lambda(h)\left(g_{i}-g_{j}\right)\right\|_{2}>$ $\sqrt{ } 2 \cdot 1 / 2$ for $i \neq j$; but then $\left\|\lambda(k)\left(g_{i}-g_{j}\right)\right\|_{2}>\sqrt{ } 2 \cdot 1 / 2-\| \lambda(k)-$ $\lambda(h)\|\cdot\| g_{i}-g_{j} \|_{2}>1 / 2(\sqrt{ } 2-1)$. It is now clear that $\lambda(k)$ cannot be compact, since $\left\{\lambda(k) g_{i}\right\}$ can have no cluster point in $L^{2}(G)$ and so is not relatively compact.

In particular when $G$ is non-compact separable abelian, $C^{*}(G$, $\left.G^{\sim}\right)$ is an extension of $\mathscr{K}^{\sim}\left(L^{2}(G)\right)$ with triple $\left(G^{\wedge},\left(G^{\wedge}\right)^{\sim}, 0\right)$ when $G$ is not discrete, and $\left(G^{\wedge}, G^{\wedge}, 0\right)$ when $G$ is discrete (since in that case the associated homomorphism of $C^{*}(G)$ into $\mathscr{L}\left(L^{2}(G)\right) / \mathscr{K}\left(L^{2}(G)\right)$ is unit preserving). This, together with Proposition 7 and the earlier remarks on end theory, completely characterizes the $C^{*}$ algebras of $(G, X)$ such that $X$ has a dense free orbit with complement a totally disconnected set of fixed points and $G$ is separable compactly generated abelian. We note that Voiculescu's recent generalization [29] of the Weyl-von Neumann theorem implies that $C^{*}\left(G, G^{\sim}\right)$ is determined (whenever $G$ is separable, noncompact, and amenable) as the unique essential split extension of $\mathscr{L}\left(L^{2}(G)\right)$ by $C^{*}(G)$. (Of course this really describes $C^{*}\left(G, G^{\sim}\right.$ ) only when $C^{*}(G)$ is known.)

2. Transformation groups with two orbit types. In this section we consider certain actions with infinitely many orbits but only a small number of orbit types. Our first goal (Proposition 11) is to show that in certain cases we can reduce to the study of simpler transformation groups.

Lemma 9. Let $Z$ be an open G-invariant subset of $X$, and $\pi$ a proper (i.e., inverse images of compact sets are compact) G-equivariant continuous map of $Z$ onto a locally compact Hausdorff $G$-space $Y$. Then there is a continuous homomorphism $\pi^{*}$ of $C^{*}(G, Y)$ into $C^{*}(G, X)$ such that $\pi^{*}(k \cdot f)=k \cdot(f \circ \pi)^{X}$ for all $k \in$ $C^{*}(G), f \in C_{\infty}(Y)$. If $G$ is amenable this homomorphism is injective. 
Proof. A slight modification of the argument for Lemma 1 (i) yields the first statement. To prove the second we invoke [26, p. 28 ] to see that (in the notation of that reference) the representation $L=\sum_{\rho}$ Ind $\rho$ of $C^{*}\left(G, Y\right.$ ) (the sum being taken over all $\rho \in C_{\infty}(Y)^{\wedge}$ ) is faithful, when $G$ is amenable. Since $C_{\infty}(Y)$ is naturally embedded as a subalgebra of $C_{\infty}(X)$, we can find (by $[8,2.10 .2]$ ) for each $\rho$ a representation $\rho^{\prime}$ of $C_{\infty}(X)$ such that $\left.o^{\prime}\right|_{C_{\infty}(Y)}$ strictly contains $\rho$. Then Ind $\rho^{\prime}$ restricted to $C^{*}(G, Y)$ strictly contains Ind $\rho$, and therefore $\sum_{\rho}$ Ind $\rho^{\prime}$ is a representation of $C^{*}(G, X)$ whose restriction to $C^{*}(G, Y)$ strictly contains $L$ and so is faithful. Since this representation of $C^{*}(G, Y)$ factors through $\pi^{*}$ the latter map must be injective.

We will use this lemma to identify $C^{*}(G, Y)$ with a subalgebra of $C^{*}(G, X)$ whenever there is no ambiguity in the choice of $\pi$.

REMARK. It can be shown that the last statement of the lemma is false when $G$ is not assumed amenable. In fact, as was pointed out to me by Jonathan Rosenberg, we can take $G=S L_{2}(R)$ and $X=G / H$ where $H$ is the subgroup of upper triangular matrices in $G$; the fact that not every representation of $G$ is weakly contained in a representation induced from $H$ implies that the natural homomorphism of $C^{*}\left(G\right.$, point) into $C^{*}(G, G / H)$ is not one-to-one.

The following lemma is a slight refinement of $[8,11.5 .3]$.

Lemma 10. Let $\mathfrak{A}=(A(t), \Theta)$ be a continuous field of $C^{*}$-algebras on the locally compact Hausdorff space $T, A$ the $C^{*}$-algebra defined by $\mathfrak{A}$, and $B \subseteq C$ two sub-C*-algebras of $A$. Suppose that for all $t_{1}, t_{2} \in T, \varepsilon>0$, and $c \in C$ there exists $b \in B$ with $\left\|b\left(t_{i}\right)-c\left(t_{i}\right)\right\|<\varepsilon$ for $i=1,2$. Then $B=C$.

Proof. The result follows from an easy modification of the proof of $[8,11.5 .3]$ once we observe (using the Krein extension theorem and the weak compactness of the closure $P(A)^{-}$of the set of pure states of $A$ ) that any $f_{0} \in P(C)^{-}$extends to an $f \in P(A)^{-}$, and hence "lives on" some $A(t)$. (It can also be deduced from [11. Theorem 1.4].)

Proposition 11. Let $(G, W)$ and $(G, Y)$ be transformation groups such that the action of $G$ on $Y$ is trivial. Give $X=Y \times W$ the "diagonal" G-action: $s \cdot(y, w)=(y, s \cdot w)$ for $y \in Y, w \in W$, and $s \in G$.

(i) There is an isomorphism $\eta$ of $C^{*}(G, X)$ onto $A=C_{\infty}(Y$, 
$\left.C^{*}(G, W)\right) \cong C_{\infty}(Y) \otimes C^{*}(G, W)$ such that $\eta(k \cdot f)(y)=k \cdot f(y, \cdot)$ for $k \in C^{*}(G), f \in C_{\infty}(Y \times W)$ (where $f(y, \cdot)$ denotes the function $w \mapsto$ $f(y, w)$ in $\left.C_{\infty}(W)\right)$.

(ii) Assume in addition that $G$ is amenable, and let $\pi$ be a continuous proper G-equivariant map of an open G-invariant subset $Z$ of $X$ onto a locally compact Hausdorff $G$-space $T$. Then $C^{*}(G, T)$ is identified (via $\eta$ ) with the subalgebra $C=\left\{\psi \in A,\left(\psi\left(y_{1}\right), \psi\left(y_{2}\right)\right) \in\right.$ $C^{*}\left(G, T_{y_{1}, y_{2}}\right)$ for all $\left.y_{1}, y_{2} \in Y\right\}$, where $T_{y_{1}, y_{2}}$ denotes the subspace $\pi\left(\left(\left\{y_{1}, y_{2}\right\} \times W\right) \cap Z\right)$ of $T$ and $C^{*}\left(G, T_{y_{1}, y_{2}}\right)$ is regarded as a subalgebra of $C^{*}\left(G,\left\{y_{1}, y_{2}\right\} \times W\right) \cong C^{*}(G, W) \times C^{*}(G, W)$ by means of Lemma 9.

Proof. (i) We see easily, using $C_{\infty}(X) \cong C_{\infty}(Y) \otimes C_{\infty}(W)$, that representations of $C^{*}(G, X)$ are in one-to-one correspondence with triples $(V, M, N)$ where $V$ is a representation of $G, M$ is a representation of $C_{\infty}(W)$, and $N$ is a representation of $C_{\infty}(Y)$ such that the images of $M$ and $N$ commute, the images of $V$ and $N$ commute, and $V(s) M(f) V\left(s^{-1}\right)=M(s f)$ for all $s \in G, f \in C_{\infty}(W)$. In other words representations $L$ of $C^{*}(G, X)$ correspond to pairs $\left(L_{0}, N\right)$ where $L_{0}$ is a representation of $C^{*}(G, W)$ and $N$ is a representation of $C_{\infty}(Y)$ such that the images of $L_{0}$ and $N$ commute; furthermore $L\left(C^{*}(G, X)\right)$ is generated by the images of $L_{0}$ and $N$. It then follows from the discussion on pp. 158-159 of [16] that $C^{*}(G, X)$ is isomorphic to the "maximal tensor product" of $C_{\infty}(Y)$ and $C^{*}(G, W)$; since $C_{\infty}(Y)$ is nuclear [16], this is the same as the minimal tensor product, which is isomorphic to $C_{\infty}\left(Y, C^{*}(G, W)\right)([23, \mathrm{pp} .59-60])$ (One can also see this directly from [28, Theorem 1].) It may easily be checked that this isomorphism has the desired effect on elements of the form $k \cdot f$ for $k \in C^{*}(G)$ and $f \in C_{\infty}(X)$.

(ii) Recall that $C^{*}(G, T)$ is the sub-C*-algebra of $C^{*}(G, X)$ generated by elements of the form $k \cdot f$ where $k$ is in $C^{*}(G)$, and $f$ in $C_{\infty}(Z)$ "factors through" $T$. Given such an element we have, for $y_{1}, y_{2}$ in $Y$, that $\left(\eta(k \cdot f)\left(y_{1}\right), \eta(k \cdot f)\left(y_{2}\right)\right)=\left(k \cdot f\left(y_{1}, \cdot\right), k \cdot f\left(y_{2}, \cdot\right)\right)$. Clearly $\left(f\left(y_{1}, \cdot\right), f\left(y_{2}, \cdot\right)\right)$ regarded as a function on $\left(\left\{y_{1}, y_{2}\right\} \times W\right) \cap Z$ factors through $T_{y_{1} y_{2}}$ and so $\eta\left(C^{*}(G, T)\right) \subseteq C$. On the other hand given $g$ in $C_{\infty}\left(T_{y_{1}, y_{2}}\right)$ we can, regarding $g$ as a function on $\left(\left\{y_{1}, y_{2}\right\} \times\right.$ $W) \cap Z$, extend it to a function $f$ in $C_{\infty}(Z)$; then with $\psi=\eta(k \cdot f)$ the above calculation shows $\left(\psi\left(y_{1}\right), \psi\left(y_{2}\right)\right)=k \cdot g$. (Here $k$ can be any element of $C^{*}(G)$.) Since the elements $k \cdot g$ generate $C^{*}\left(G, T_{y_{1}, y_{2}}\right)$ and since $C$ is obviously a sub- $C^{*}$-algebra of $A$, it is clear from Lemma 10 (taking $B=\eta\left(C^{*}(G, T)\right), T=Y$, and $\mathfrak{A}=$ the trivial continuous field of $C^{*}$-algebras defined by the decomposition of $A$ as $C_{\infty}(Y) \otimes$ $C^{*}(G, W)$-cf. $\left.[8,10.5]\right)$ that $C=\eta\left(C^{*}(G, T)\right)$. 
This proposition can be used to derive a result of Rosenberg concerning the $C^{*}$-algebras of a family of solvable Lie groups. Namely, let $H$ be a semidirect product of $\boldsymbol{R}$ with $\boldsymbol{R}^{m}$ such that the roots of the action of $\boldsymbol{R}$ on $\boldsymbol{R}^{m}$ all have non-zero real parts of the same sign. Then as was observed in [22], $C^{*}(H)$ is isomorphic to $C^{*}(G, T)$ where $G=R$ acts on $T=R^{m}$ via $r \cdot v=e^{r} v$ for $r \in R, v \in$ $\boldsymbol{R}^{m}$. It is easy to see that we may identify $T$ with the "proper quotient" of $S^{m-1} \times X_{1}$ (where $X_{1}$ is taken as in Proposition 7) obtained by collapsing $S^{m-1} \times\{\infty\}$ to a single point. Hence the preceding proposition applies, with $T_{y_{1}, y_{2}}$ the quotient space of $\left\{y_{1}, y_{2}\right\} \times$ $X_{1}$ obtained by identifying $\left(y_{1}, \infty\right)$ with $\left(y_{2}, \infty\right)$, for all pairs $\left\{y_{1}, y_{2}\right\}$ in $Y=S^{m-1}$. To compute $D=C^{*}\left(G, T_{y_{1}, y_{2}}\right)$ (which incidentally is just the $C^{*}$-algebra of the proper real " $a x+b$ " group) as a subalgebra of $F=C^{*}\left(G,\left\{y_{1}, y_{2}\right\} \times X_{1}\right) \simeq C^{*}\left(G, X_{1}\right) \times C^{*}\left(G, X_{1}\right)$ we observe, from the explicit formula given for the embedding $\pi^{*}$ in Lemma 9, and from Lemma 1 , that $\pi^{*}(D)$ contains the ideal $I=C^{*}(G, G) \times$ $C^{*}(G, G)$, and that $\pi^{*}(D) / I$ is the "diagonal subalgebra" of

$$
F / I=C^{*}\left(G,\left\{\left(y_{1}, \infty\right),\left(y_{2}, \infty\right)\right\}\right) \cong C^{*}(G) \times C^{*}(G) .
$$

Hence

$$
C^{*}\left(G, T_{y_{1}, y_{2}}\right)=\left\{\left(e_{1}, e_{2}\right) \in C^{*}\left(G, X_{1}\right) \times C^{*}\left(G, X_{1}\right) \mid \beta\left(e_{1}\right)=\beta\left(e_{2}\right)\right\},
$$

where $\beta=\beta_{\{\infty\}}$ denotes the canonical homomorphism of $C^{*}\left(G, X_{1}\right)$ onto $C^{*}(G,\{\infty\})$. Therefore by Proposition $11 C^{*}(H)$ is isomorphic to the algebra

$$
C=\left\{\psi \in C_{\infty}\left(S^{m-1}, C^{*}\left(G, X_{1}\right)\right) \mid \beta(\psi(y)) \text { is constant in } y \in S^{m-1}\right\} .
$$

One can easily verify that this description is equivalent to that given in Propositions 3, 4, 5 of [22], i.e., that $C^{*}(H)$ is an extension of $C_{\infty}\left(S^{m-1}, \mathscr{K}\left(L^{2}(\boldsymbol{R})\right)\right)$ by $C_{\infty}(\boldsymbol{R})$ with the index constant (in $y \in S^{m-1}$ ) and equal to 1 .

We remark without giving details that a similar argument provides the structure of the $C^{*}$-algebras of the complex and $p$-adic analogues of the above groups. The key feature of the semi-direct product groups treatable by this method is that, for the associated transformation group $(G, X), X$ has a single fixed point whose complement is a trivial $G$-principal fiber bundle over its orbit space, with the latter being compact. We proceed now to investigate a slightly more general class of transformation groups to which the method can be applied. Namely, throughout the remainder of this section we assume the following conditions on $(G, X)$ :

(i ) $G$ is compactly generated 
(ii) the set $Y$ of $G$-fixed points of $X$ totally disconnected

(iii) $Z=X \backslash Y$ is dense in $X$, and $Z / G$ is compact Hausdorff

(*) (iv) there is a $G$-isomorphism $(Z / G) \times G \rightarrow Z$ (where $(Z / G) \times G$ is given the "diagonal action" coming from the action by left translation on $G$ and the trivial action on $Z / G$ ).

Our first goal is to show that it is possible to "build up" such a $(G, X)$ from copies of a transformation group having a single dense orbit via the construction of Proposition 11.

As in Lemma 4 we will let $E$ denote the compact space obtained by adjoining to $G$ its set $\xi$ of ends. $\varphi$ will denote a $G$-isomorphism $(Z / G) \times G \rightarrow Z$.

Proposition 12. With $(G, X)$ as above there is a continuous proper G-equivariant map $\pi$ of an open subset $\mathscr{W}$ of $(Z / G) \times E$ onto $X$ such that $\mathscr{W} \supseteqq(Z / G) \times G$ and $\left.\pi\right|_{(Z / G) \times G}=\varphi$.

Proof. If $X$ is not compact, note that $X^{\sim}$ (with the natural $G$ action) satisfies the hypotheses (*). We shall prove that for $(G, X)$ satisfying $(*)$, and with $X$ compact, we may find $\pi$ as in the proposition and defined on all of $(Z / G) \times E$; for non-compact $X$ this result applied to $X^{\sim}$ yields the proposition, since we can then take $\mathscr{W}^{-}=\pi^{-1}(X)$.

The construction of $\pi$ goes as follows: given $W \in Z / G$, we identify $G$ with $W$ via $\varphi_{W}: s \mapsto \varphi((W, s))$. Then $\left(G, W^{-}\right)$satisfies the hypotheses of Lemma 4 , hence there is a continuous proper map $\pi_{W}: E \mapsto X$ extending $\varphi_{W}$. Now define $\pi$ by $\pi(W, t)=\pi_{W}(t)$ for all $W \in Z / G, t \in E$.

We prove $\pi$ is continuous; the other properties claimed for it will then be obvious. Continuity at points in $(Z / G) \times G$ is clear, so we must show for any $(W, \varepsilon)$ in $Z / G \times \xi$ and any neighborhood $V$ of $y=\pi(W, \varepsilon)$ that $\pi^{-1}(V)$ is a neighborhood of $(W, \varepsilon)$. We may assume that $V$ is compact, that $V \cap Y$ is open in $Y$ (since $Y$ is totally disconnected), and that $V^{\circ} \cap Y=V \cap Y$, where $V^{\circ}$ is the interior of $V$. Let $U$ be a compact symmetric neighborhood of the identity whose interior generates $G$. Then an easy compactness argument shows that we can find an open neighborhood $V_{1}$ of $y$ such that $V_{1} \cap Y=V \cap Y$ and $U^{2} \cdot V_{1} \subseteq V$. The subset $K=V \backslash V_{1}$ of $Z$ is compact, so (denoting by $p_{1}$ and $p_{2}$ the projections of $(Z / G) \times$ $G$ onto the first and second factors, respectively) $C=p_{2}\left(\varphi^{-1}(K)\right)$ is a compact subset of $G$. Now let $T(s)$ denote, for any $s \in G$ and $T \subseteq X$, the subset $p_{1}\left(\pi^{-1}(T) \cap(Z / G \times\{s\})\right)$ of $Z / G$. 
Choose $Q \in \varepsilon$ disjoint from $C$ and " $U$-connected." (See [20, pp. 19-21] for definitions and the proof that $Q$ can be taken $U$-connected.) Then, for each $s$ in $Q$ and $t$ in $U, V(s)=t V_{2}(s)=V_{1}(s)$ (since $s \notin C$ ), where $V_{2}=U V_{1}$. The $U$-connectedness of $Q$ together with the fact that $t V_{2}(s)=V_{2}\left(t^{-1} s\right)$ now imply that $V_{2}\left(s_{1}\right)=V_{2}\left(s_{2}\right)$ for all $s_{1}, s_{2} \in Q$. As $V_{1}$ is open so is $V_{1}(s)$, and since $\pi(W, \varepsilon)=y$ and $Q \in \varepsilon$ we must have $W \in V_{1}(s)$ (from the way $\pi$ was defined). A simple argument now shows that $V_{1}(s) \times\left(Q \cup\left\{\varepsilon^{\prime} \in \xi: Q \in \varepsilon^{\prime}\right\}\right)$ is a neighborhood of ( $W$, $\varepsilon$ ) which is sent into $V$ by $\pi$. Hence $\pi$ is continuous at $(W, \varepsilon)$, as was to be proved.

This result tells us that when we have $(G, X)$ satisfying (*) we may apply Proposition 11 to the problem of computing $C^{*}(G, X)$. Of course, a satisfactory solution is possible only when $C^{*}(G, E)$ is known. When $G$ is separable compactly generated abelian we can use the results of $\S 1$ to deduce the theorem below; the argument is similar to that given above in computing the structure of the $C^{*}$-algebras of Rosenberg's Lie groups, and so is omitted. The notation is that of the preceding proposition.

THEOREM 13. Let $(G, X)$ satisfy (*), and suppose $G=\boldsymbol{Z}^{n} \times \boldsymbol{R}^{m} \times$ $K$ where $K$ is a separable compact abelian group. (i) If $G$ has one end (i.e., if $n+m \geqq 2$ ), denoted $\infty$, let $\beta$ be the canonical homomorphism of $C^{*}(G, E)$ onto $C^{*}(G) \cong C^{*}(G,\{\infty\}$ ) (using the construction of Lemma 1). Then

$$
\begin{aligned}
& C^{*}(G, X) \cong\left\{\psi \in C_{\infty}\left(Z / G, C^{*}(G, E)\right) \mid \beta(\psi(z))=0 \text { if }(z, \infty) \notin \mathscr{W},\right. \\
& \text { and } \beta\left(\psi\left(z_{1}\right)\right)=\beta\left(\psi\left(z_{2}\right)\right) \text { for all pairs }\left\{z_{1}, z_{2}\right\} \text { such that } \\
& \left.\pi\left(z_{1}, \infty\right)=\pi\left(z_{2}, \infty\right)\right\} .
\end{aligned}
$$

Here $C^{*}(G, E)$ is a split extension of $C^{*}(G, G) \cong \mathscr{K}\left(L^{2}(G)\right)$ by $C^{*}(G)$.

(ii) If $G$ has two ends, denoted $\infty_{-1}$ and $\infty_{+1}$, let $\tau$ denote the automorphism of $C^{*}(G)$ induced by the automorphism $(r, t) \mapsto(-r, t)$ of $G$ (recall $G=\boldsymbol{Z} \times K$ or $\boldsymbol{R} \times K$ ), and let $\beta_{i}$ be the homomorphism of $C^{*}(G, E)$ onto $C^{*}(G) \cong C^{*}\left(G,\left\{\infty_{i}\right\}\right)$ for $i= \pm 1$. Then

$$
\begin{aligned}
& C^{*}(G, X) \cong\left\{\psi \in C_{\infty}\left(Z / G, C^{*}(G, E)\right) \mid \beta_{i}(\psi(z))=0 \text { if }\left(z, \infty_{i}\right) \notin \mathscr{W},\right. \\
& \beta_{i}\left(\psi\left(z_{1}\right)\right)=\beta_{i}\left(\psi\left(z_{2}\right)\right) \text { if } \pi\left(z_{1}, \infty_{i}\right)=\pi\left(z_{2}, \infty_{i}\right) \text {, and } \\
& \beta_{1}\left(\psi\left(z_{1}\right)\right)=\tau\left(\beta_{-1}\left(\psi\left(z_{2}\right)\right)\right) \text { if } \pi\left(z_{1}, \infty_{+1}\right)=\pi\left(z_{2}, \infty_{-1}\right), \\
& \text { for all } \left.z, z_{1}, z_{2} \in Z / G \text { and } i= \pm 1\right\} .
\end{aligned}
$$

Here the structure of $C^{*}(G, E)$ is given in Proposition 7 (as $(G, E$ ) is isomorphic to the $(G, X)$ of that proposition). 
The reason for introducing the automorphism $\tau$ in part (ii) of the above proposition is that $G$ acts "in opposite directions" near the two ends $\infty_{ \pm 1}$. We can illustrate the significance of this by considering the example $C^{*}\left(\boldsymbol{R}, \boldsymbol{R}^{\sim}\right)$. By Proposition 8 this algebra is a split extension of $\mathscr{K}\left(L^{2}(\boldsymbol{R})\right)$, which would seem to contradict the fact that by the above proposition it sits as a sub-algebra of the non-split extension $C^{*}(\boldsymbol{R},[-\infty, \infty])$; the point, however, is that the image of $C^{*}\left(\boldsymbol{R}, \boldsymbol{R}^{\sim}\right)$ in $C^{*}(\boldsymbol{R},\{-\infty, \infty\}) \simeq C^{*}(\boldsymbol{R}) \times C^{*}(\boldsymbol{R})$ (after dividing out by the ideal $\left.C^{*}(\boldsymbol{R}, \boldsymbol{R})\right)$ is the "skew diagonal" $\{(\tau(\alpha)$, a) $\left.\mid a \in C^{*}(\boldsymbol{R})\right\}$ rather than the diagonal, and it is precisely this skewness that "untwists" the extension so as to make it split.

3. Transformation groups with continuous trace $C^{*}$-algebra. In this final section we consider free actions only. Our goal is to find a necessary and sufficient condition for the associated $C^{*}$-algebra to be a continuous trace algebra, and, when it is, to investigate more carefully the structure of the algebra. Rather surprisingly, it turns out that the continuous trace algebras $A$ which arise always have the property that, when it is defined, the associated element (cf. [9]) of the Čech cohomology group $H^{3}\left(A^{\wedge}, Z\right)$ is 0 ; intuitively, this means that (at least when $G$ is not finite) $C^{*}(G, X)$ is never "twisted" over its spectrum, even when $X$ is "twisted" over $X / G$.

We begin by generalizing slightly a concept familiar from the theory of dynamical systems (cf. [18, p. 22].)

Definition. A subset $U$ of $X$ is wandering if $\{s \in G \mid s U \cap U \neq$ $\varnothing\}$ is relatively compact in $G$.

The relevant condition on $(G, X)$ for our purposes is then that every compact subset of $X$ be wandering. (Note that this is always satisfied when $X$ is a $G$-principal fiber bundle over $X / G$.) First we show what this condition implies for the structure of $C^{*}(G, X)$.

THEOREM 14. Assume that $G$ acts freely on $X$ and that compact sets in $X$ are wandering. Then $X / G$ is Hausdorff, and there is a continuous field $\mathscr{H}=((H(x)), \Gamma)$ of Hilbert spaces on $X / G$ such that $C^{*}(G, X)$ is isomorphic to the $C^{*}$-algebra defined by the continuous field of elementary $C^{*}$-algebras associated to $\mathscr{H}$. (cf. [8, 10.4.1 and 10.7.2]). In particular $C^{*}(G, X)$ is a continuous trace $C^{*}$-algebra with spectrum homeomorphic to $X / G$.

Proof. We begin by showing that for each $x$ in $X$ the map $\varphi_{x}: s \mapsto s x$ of $G$ into $X$ is a homeomorphism onto its image: Let $U$ be an open relatively compact neighborhood of $x$. Then $\varphi_{x}^{-1}(U)$ is open in $G$, and since it is contained in the set $\{s \in G: s U \cap U \neq \varnothing\}$ 
it is relatively compact. Hence since $\varphi_{x}$ takes compact sets homeomorphically onto their images, $\left.\varphi_{x}\right|_{\varphi x^{-1}(U)}$ is a homeomorphism onto $U \cap G x$. It follows that the image of any neighborhood of the identity of $G$ under the map $\varphi_{x}$ is a neighborhood of $x$ in $G x$. By homogeneity $\varphi_{x}$ is open onto its image, and hence a homeomorphism.

Next we show that $X / G$ is Hausdorff: If it were not we could find a net $\left(\mathscr{W}_{\alpha}\right)$ in $X / G$ converging to two distinct points $Y_{1}$ and $Y_{2}$, and thus (by openness of the projection of $X$ onto $X / G$ ) nets $s_{\alpha}$ in $G$ and $x_{\alpha}$ in $X$ with $x_{\alpha}$ converging to $y_{1}$ and $s_{\alpha} x_{\alpha}$ to $y_{2}$, where $y_{1} \in Y_{1}$ $y_{2} \in Y_{2}$. Choose compact neighborhoods $U_{1}$ and $U_{2}$ of $y_{1}$ and $y_{2}$; the fact that $U_{1} \cup U_{2}$ is wandering then implies that the $s_{\alpha}$ lie in a compact subset of $G$ for large $\alpha$. Passing to a subnet we may assume the $s_{\alpha}$ converge to some element $s$ in $G$. But then joint continuity of the action and Hausdorffness of $X$ imply that $y_{2}=s y_{1}$, so $Y_{1}=Y_{2}$, a contradiction. Hence $X / G$ is Hausdorff. In particular each orbit is closed.

Now let $\mu$ be a fixed right Haar measure on $G$. For each $W$ in $X / G$ we define a measure $\mu_{W}$ on $W$ by choosing $x$ in $W$ and letting $\mu_{W}$ be the image of $\mu$ under the map $\varphi_{x}: G \rightarrow W$. A simple computation shows that $\mu_{W}$ is independent of the choice of $x$, so $H_{W}=L^{2}\left(W, \mu_{W}\right)$ is well defined. Each $f$ in $C_{c}(X)$ defines by restriction an element of $C_{c}(W)$ and hence of $H_{W}$; in this way we associate to each such $f$ a field of vectors $v_{f}$ over $X / G$. Now $\left\|v_{f}(W)\right\|_{2}=$ $\left(\int\left|f \circ \varphi_{x}\right|^{2} d \mu(s)\right)^{1 / 2}$ for any $x$ in $W$. The wandering hypothesis implies that, given a compact neighborhood $U$ of $x$, there is a compact subset of $G$ containing the supports of all $f \circ \varphi_{y}$ for $y$ in $U$, and it is easy to see from this that the right hand side of the preceding equation must vary continuously in $x$. It follows that the left hand side varies continuously in $W$. Thus hypothesis (iii) of $[8,10.1 .2]$ is satisfied by $\Gamma_{0}=\left\{v_{f}: f \in C_{c}(X)\right\}$; hypotheses (i) and (ii) are obvious for $\Gamma_{0}$, so by $[8,10.2 .3]$ there is a unique subset of $\Pi_{W \in X / G} H_{W}$ containing $\Gamma_{0}$ and defining a continuous field of Hilbert spaces over $X / G$. We let $\mathscr{A}=(A(W), \Theta)$ denote the associated field of elementary $C^{*}$-algebras $([8,10.7 .2])$, and $A$ the $C^{*}$-algebra defined by $\mathfrak{A}$.

For each $W \in X / G$ define a representation $L_{W}$ of $C^{*}(G, X)$ on $L^{2}\left(W, \mu_{W}\right)$ by letting $C_{\infty}(X)$ act by multiplication of functions, and $G$ act by $\left(V_{W}(s) h\right)(x)=h\left(s^{-1} x\right) \Delta_{G}(s)^{1 / 2}$ for $h \in L^{2}(W)$ and $s \in G$. Then for each $x$ in $X$ the unitary transformation $S_{x}: L^{2}(W) \rightarrow L^{2}(G, \mu)$, $h \mapsto h \circ \varphi_{x}$ intertwines $L_{W}$ with a representation equivalent to the representation of Lemma 3. Hence $L_{W}\left(C^{*}(G, X)\right)=\mathscr{K}\left(L^{2}(W)\right)$.

We wish to show, more precisely, that $C^{*}(G, X)$ is isomorphic to $A$. Let, therefore, $T_{b}$ for any $b \in C^{*}(G, X)$ denote the field $\left(L_{W}(b)\right)_{W \in X / G}$ over $X / G$. By definition of $\mathfrak{A}, T_{b}$ will be in $\Theta$ if for 
any $W \in X / G$ and $\varepsilon>0$ we can find $f_{i}, g_{i} \in C_{c}(X)(i=1, \cdots, n)$, such that for some neighborhood $\mathscr{U}$ of $W$,

$$
\left\|L_{W^{\prime}}(b)-\sum_{i=1}^{n} \theta_{f_{i}, q_{i}}\left(W^{\prime}\right)\right\|<\varepsilon \text { for all } W^{\prime} \in \mathscr{U}
$$

where $\theta_{f_{i}, g_{i}}$ is defined (see $[8,10.7 .2$ and 10.6.1]) to be the following field of rank one operators: $\theta_{f_{i} g_{i}}\left(W_{0}\right)(h)=\left(h, g_{i}\right)_{L^{2}\left(W_{0}\right)} f_{i}$, for all $W_{0} \in$ $X / G$ and $h \in L^{2}\left(W_{0}\right)$.

Observe that it is sufficient to check this condition for $b$ in $C^{*}(G, X)$ of the form $k \cdot f$, where $k \in C^{*}(G)$ and $f \in C_{c}(X)$, since these generate $C^{*}(G, X)$ as a $C^{*}$-algebra. Given such $a b$, and given $W \in$ $X / G$ and $\varepsilon>0$, we use the fact that $L_{W}(b)$ is compact, and hence can be approximated by finite rank operators, to find $f_{i}, g_{i}, i=1, \cdots, n$ such that $\left\|L_{W}(b)-\sum_{i=1}^{n} \theta_{f_{i} g_{i}}(W)\right\|<\varepsilon / 2$. Now, arguing as in the demonstration that any $\left\|v_{f}(W)\right\|$ varies continuously in $W$, we see that the "pull-backs" $S_{x} M_{W(x)}(f) S_{x}^{-1}$ and $S_{x} \theta_{f_{i} g_{i}}(W(x)) S_{x}^{-1}$ (where $W(x)$ denotes the orbit of $x$ ) vary continuously in $x$. Since $S_{x} V_{W(x)}(k) S_{x}^{-1}$ is constant (being the image of $k$ under the regular representation) it is clear that $\left\|L_{W^{\prime}}(b)-\sum_{i=1}^{n} \theta_{f_{i} g_{i}}\left(W^{\prime}\right)\right\|$ varies continuously in $W^{\prime}$ and so is less than $\varepsilon$ in some neighborhood of $W$.

Now observe that, for $b=k \cdot f$ as above, $\left\|L_{W}(b)\right\|$ is zero outside the image in $X / G$ of the compact set supporting $f$. This together with the preceding paragraph implies that $b \mapsto T_{b}$ defines a homomorphism of $C^{*}(G, X)$ into the $C^{*}$-algebra $A$ defined by $\mathfrak{A}$; it remains to check that this homomorphism is injective and surjective.

We see from the fact that the "irreducible" closed $G$-invariant subsets are precisely the orbits, together with Lemma 1 (ii) and (iii) that any irreducible representation of $C^{*}(G, X)$ "factors through" $C^{*}(G, W)$ for some orbit $W$, and so (by Lemma 3 and $[8,4.1 .5]$ ) is equivalent to $L_{W}$. Hence for any $b \in C^{*}(G, X)$ we have $\|b\|=$ $\sup _{W \in X / G}\left\|L_{W}(b)\right\|$, which agrees with its norm as an element of $A$. Therefore the homomorphism is isometric, hence injective.

To see that it is surjective we invoke $[8,10.5 .3]$. Thus given $W_{1} \neq W_{2} \in X / G$, let $Z_{1}$ and $Z_{2}$ be the inverse images in $X$ of disjoint open neighborhoods of $W_{1}$ and $W_{2}$ in $X / G$. By Lemmas 1 and 3, $C^{*}\left(G, Z_{1}\right)$ and $C^{*}\left(G, Z_{2}\right)$ are disjoint ideals of $C^{*}(G, X)$ such that $L_{W_{i}}\left(C^{*}\left(G, Z_{i}\right)\right)=\mathscr{K}\left(L^{2}\left(W_{i}\right)\right)$ for $i=1,2$, while $L_{W_{1}}\left(C^{*}\left(G, Z_{2}\right)\right)=(0)=$ $L_{W_{2}}\left(C^{*}\left(G, Z_{1}\right)\right)$. Therefore given any $R_{1} \in \mathscr{K}\left(L^{2}\left(W_{1}\right)\right), R_{2} \in \mathscr{K}\left(L^{2}\left(W_{2}\right)\right)$ we can find $b_{i} \in C^{*}\left(G, Z_{i}\right)$ so that $L_{W_{i}}\left(b_{i}\right)=R_{i}$, and then putting $b=$ $b_{1}+b_{2}$ we have $L_{W_{i}}(b)=R_{i}$. Thus by $[8,10.5 .3] C^{*}(G, X)$ maps surjectively to $A$.

That $C^{*}(G, X)$ is a continuous trace $C^{*}$-algebra with spectrum $X / G$ we may now deduce from $[8,10.7 .7,10.5 .8$, and 10.5.2]. 
Corollary 15. Let $(G, X)$ be as in the theorem. Assume in addition that $G$ is not finite, $X / G$ is paracompact (this is always true of course if $X$ is second countable), and that either (i) $X / G$ has finite covering dimension and $G$ is separable, or (ii) $X$ is a principal G-fiber bundle over $X / G$. Then $C^{*}(G, X)$ is isomorphic to $C_{\infty}\left(X / G, \mathscr{K}\left(L^{2}(G)\right)\right)$.

Proof. If $X$ is a $G$-fiber bundle over $X / G$ it is clear that the continuous field of Hilbert spaces constructed in Theorem 14 is locally trivial, while if $X / G$ has finite dimension, continuous fields of separable infinite dimensional Hilbert spaces over it are always locally trivial by $[8,10.8 .7]$. In either case we conclude by [9] that the field is in fact trivial. (Nonfiniteness of $G$ implies each $L^{2}(W)$ is infinite dimensional.) The desired result now follows from the obvious fact that the continuous field of $C^{*}$-algebras associated to a trivial field of Hilbert spaces is itself trivial.

As a consequence of the above corollary we see that it is possible to have $C^{*}\left(G, X_{1}\right) \cong C^{*}\left(G, X_{2}\right)$ when $X_{1}$ and $X_{2}$ are not homeomorphic (take $G=Z, X_{1}=R, X_{2}=Z \times T$, with $Z$ acting by translation in each case).

It is perhaps worth pointing out the consequences of the above corollary for actions of compact groups. Such actions always have the property that compact sets are wandering; hence if the action is free we know that the associated $C^{*}$-algebra is a continuous trace algebra. Gleason has proved [12] that if $G$ is a compact Lie group acting freely on a completely regular space $X$ then $X$ is a fiber bundle over $X / G$. Thus when $X / G$ (or equivalently $X$ ) is paracompact we can conclude that (at least when $G$ is not finite) the structure of $C^{*}(G, X)$ depends only on the topology of $X / G$.

We turn now to a converse of the above theorem in the case where $G$ and $X$ are second countable. We will need some facts about induced representations of $C^{*}(G, X)$, which may be found in [10] or [27].

Lemma 16. Let $G$ and $X$ be second countable, with $G$ acting freely on $X$, and assume that $A=C^{*}(G, X)$ is type $I$. Then the map $X \rightarrow \operatorname{Rep} A$ which sends $x$ to Ind $\chi_{x}$ (the representation induced from the character $\chi_{x}$ of $C_{\infty}(X)$ determined by $x$ ) induces a homeomorphism of $X / G$ with $A^{\wedge}$. The map of Lemma 1 (i) from open $G$-invariant subsets of $X$ to ideals of $C^{*}(G, X)$ is a bijection.

Proof. By [14] every "quasi-orbit" (in the sense of [10]) in $X$ is an orbit, so $([10,5.2])$ the restriction of any irreducible represen- 
tation of $A$ lives on an orbit. Thus by [27] any irreducible representation is equivalent to Ind $\left(\chi_{x}\right)$ for some $x$. We can now deduce easily from [10, p. 68] that the indicated map from $X$ to Rep $A$ induces a homeomorphism of $X / G$ with $A^{\wedge}$.

The second claim follows from the preceding and the fact that an ideal $I$ of $A$ is uniquely determined by the set $\mathcal{O}_{I}$ of (equivalence classes of) irreducible representations which do not annihilate it.

It will be convenient in the proof of the next theorem to work with the following realization of Ind $\chi_{x}$ on $L^{2}(G)$ : Let $V_{x}$ be the left regular representation $\lambda$ of $G$, and let $M_{x}(f)$, for $f$ in $C_{\infty}(X)$, be the multiplication operator associated to the bounded continuous function $f \circ \varphi_{x}$ on $G$ (where $\varphi_{x}$ is as in the proof of Theorem 14). Then the pair $\left(V_{x}, M_{x}\right)$ determines a representation $L_{x}$ of $C^{*}(G, X)$ equivalent to Ind $\chi_{x}$.

For the remainder of the paper we drop our convention that all ideals are to be closed.

THEOREM 17. Let $G$ and $X$ be second countable, with $G$ acting freely on $X$. Then $C^{*}(G, X)$ is a continuous trace algebra if and only if every compact subset of $X$ is wandering.

Proof. The "if" implication is a consequence of Theorem 14.

Hence assume that $A=C^{*}(G, X)$ is a continuous trace algebra. Since $A$ is then type $I$ and $A^{\wedge}$ is Hausdorff, the preceding lemma implies that $X / G$ is Hausdorff. Suppose there exists a nonwandering compact subset $K$ of $X$; then we can choose sequences $\left(x_{i}\right)$ in $K$ and $\left(s_{i}\right)$ in $G$, with the $s_{i}$ not all contained in any compact subset of $G$, but with $s_{i} x_{i}$ in $K$ for all $i$. Passing to subsequences we may assume $x_{i}$ tends to a limit $z$ in $K$, and $s_{i} x_{i}$ tends to $y \in K$. By Hausdorffness of $X / G, z$ and $y$ must lie in the same $G$ orbit; so, taking $s$ in $\mathrm{G}$ such that $z=s y$, we have $t_{i} x_{i}$ tending to $z$, where $\left(t_{i}\right)=\left(s s_{i}\right)$ is an unbounded sequence in $G$. Our aim in the remainder of the proof will be to use this "double clustering" of the orbits $G x_{i}$ at $z$ to produce a positive element $c$ in the Pedersen ideal ([19]) of $A$ for which the function $\pi \mapsto \operatorname{Tr}(\pi(c))$ is not continuous on $A^{\wedge}$, thus contradicting our hypothesis that $A$ is a continuous trace algebra (since the ideal of continuous trace elements in such an algebra is dense and hereditary, and so must contain the Pedersen ideal).

Let $f \in C_{c}(X)$ be nonnegative and real-valued with $f(z)=4$ and let $Q$ be a compact symmetric neighborhood of the identity of $G$. Then $M_{z}(f)$ is a positive operator of norm $\geqq 4$ on $L^{2}(G)$, so (since there is a bounded approximate identity for $C^{*}(G)$ consisting of 
functions in $C_{c}(G)$ whose supports shrink to the identity) we can find $k \in C_{c}(G)$ with support contained in $Q$ such that $\|k\|_{C^{*}(G)} \leqq 1$ and such that $T_{z}=L_{z}\left(k^{*} f k\right)=V_{z}(k)^{*} M_{z}(f) V_{z}(k)$ has norm $\geqq 3$.

Let $r$ be the real-valued function on $[0, \infty)$ such that $r(t)=0$ for $t \leqq 1, r(t)=t$ for $t \geqq 2$, and $r$ is linear on the interval [1,2]. Then $c=r\left(k^{*} f k\right)$ is in the Pedersen ideal $\kappa(A)$ of $A$ (cf. [19, p. 134]). Furthermore $L_{z}(c)=r\left(T_{z}\right)$ is in the Pedersen ideal of $\mathscr{K}\left(L^{2}(G)\right)$ and so $([19$, p. 133]) is a finite rank operator, say of rank $n$. Since the map $T \mapsto r(T)$ is continuous on the positive cone of $\mathscr{L}\left(L^{2}(G)\right)([17$, Lemma 1]) we may choose $\delta<1$ such that $\| r(T)-$ $\left.L_{z}(c)\right) \|<1 / n$ for each positive operator $T$ satisfying $\left\|T-T_{z}\right\|<\delta$.

For each $x$ in $X$ let $f_{x}=f \circ \varphi_{x}$. Because orbits are closed, and $\varphi_{x}$ (by standard Baire category arguments, using separability of $G$ ) is a homeomorphism, each $f_{x}$ has compact support in $G$; we let $F$ denote the support of $f_{z}$. Straightforward compactness arguments show that there is a neighborhood $U_{1}$ of $z$ such that, for each $x$ in $U_{1},\left|f_{z}(s)-f_{x}(s)\right|<\delta$ for all $s$ in $Q^{4} F$, and $f_{x}(s)=0$ for all $s$ in $Q^{4} F \backslash Q F$. Thus if we define $f_{x}^{(0)}$ for such $x$ to be equal to $f_{x}$ on $Q F$, and 0 off $Q F$, then $f_{x}^{(0)}$ is a continuous function with $\left\|f_{z}-f_{x}^{(0)}\right\|_{\infty}<$ $\delta$. Furthermore $f_{x}^{(1)}=f_{x}-f_{x}^{(0)}$ is a non-negative continuous function, and it is readily computed that $T_{x}^{(0)}=V(k)^{*} M\left(f_{x}^{(0)}\right) V(k)$ and $T_{x}^{(1)}=V(k)^{*} M\left(f_{x}^{(1)}\right) V(k)$ annihilate each other (where $V$ is the left regular representation of $G$ and $M$ represents $C_{\infty}(G)$ by multiplication of functions). Applying the functional calculus to the commutative $C^{*}$-algebra generated by $T_{x}^{(0)}$ and $T_{x}^{(1)}$ we see that $r\left(T_{x}\right)=$ $r\left(T_{x}^{(0)}\right)+r\left(T_{x}^{(1)}\right)$, where $T_{x}=T_{x}^{(0)}+T_{x}^{(1)}$ is just $L_{x}\left(k^{*} f k\right)$.

We wish to show that $c$ cannot be a continuous trace element. Thus let $U$ be any neighborhood of $z$ contained in $U_{1}$, and recall that by our choice of $z$ there exist $x_{0} \in U, s_{0} \in G$ such that $s_{0} x_{0} \in U$ but $s_{0}$ is not in the compact subset $Q^{4} F$ of $G$. Letting $R_{s_{0}} g$ denote the right translate of $g$ for any function $g$ on $G$ (i.e., $\left(R_{s_{0}} g\right)(s)=$ $g\left(s s_{0}\right)$ for all $s$ in $\left.G\right)$, we have

$$
R_{s_{0}} f_{x_{0}}^{(0)}+R_{s_{0}} f_{x_{0}}^{(1)}=R_{s_{0}} f_{x_{0}}=f_{s_{0} x_{0}}=f_{s_{0} x_{0}}^{(0)}+f_{s_{0} x_{0}}^{(1)} .
$$

Since the supports of $R_{s_{0}} f_{x_{0}}^{(0)}$ and $f_{s_{0} x_{0}}^{(0)}$ are disjoint and all terms are nonnegative, necessarily $R_{s_{0}} f_{x_{0}}^{(1)} \geqq f_{s_{0} x_{0}}^{(0)}$, so (because $\rho\left(s_{0}\right)$ commutes with $V(k)$, where $\rho$ is the right regular representation of $G$ )

$$
\rho\left(s_{0}\right) T_{x_{0}}^{(1)} \rho\left(s_{0}^{-1}\right) \geqq V(k)^{*} M\left(f_{s_{0} x_{0}}^{(0)}\right) V(k) .
$$

Taking norms we get

$$
\left\|T_{x_{0}}^{(1)}\right\| \geqq\left\|V(k)^{*} M\left(f_{z}\right) V(k)\right\|-\left\|f_{z}-f_{s_{0} x_{0}}^{(0)}\right\| \geqq\left\|T_{z}\right\|-\delta \geqq 2 .
$$

Since $r(t)=t$ for $t \geqq 2,\left\|r\left(T_{x_{0}}^{(1)}\right)\right\| \geqq 2$, and thus since the trace of 
a positive compact operator dominates its norm we conclude that $\operatorname{Tr}\left(r\left(T_{x_{0}}^{(1)}\right)\right) \geqq 2$.

Now consider $T_{x_{0}}^{(0)}$. Clearly $\left\|T_{x_{0}}^{(0)}-T_{z}\right\|<\delta$ so, by our choice of $\delta,\left\|r\left(T_{x_{0}}^{(0)}\right)-r\left(T_{z}\right)\right\|<1 / n$. Computing the trace with respect to an orthonormal basis of $L^{2}(G)$ which includes bases for the eigenspaces of the rank $n$ positive operator $r\left(T_{z}\right)$, we find

$$
\operatorname{Tr}\left(r\left(T_{x_{0}}^{(0)}\right)>\operatorname{Tr}\left(r\left(T_{z}\right)\right)-n \cdot 1 / n=\operatorname{Tr}\left(r\left(T_{z}\right)\right)-1 .\right.
$$

This combined with the conclusion of the preceding paragraph yields

$$
\operatorname{Tr}\left(r\left(T_{x_{0}}\right)\right)=\operatorname{Tr}\left(r\left(T_{x_{0}}^{(0)}\right)+r\left(T_{x_{0}}^{(1)}\right)\right)>\operatorname{Tr}\left(r\left(T_{z}\right)\right)-1+2=\operatorname{Tr}\left(r\left(T_{z}\right)\right)+1 .
$$

It follows (from the freedom in our choice of the neighborhood $U$ ) that the function $\pi \mapsto \operatorname{Tr}(\pi(c))$ on $A^{\wedge}$ cannot be continuous at $\pi=L_{z}$; as was fobserved earlier this contradicts our hypothesis that $A$ is continuous trace, and so we conclude that no non-wandering compact set can exist in $X$.

CoROLlaRY 18. Let $G$ and $X$ be second countable, with $G$ acting freely on $X$ and with $A=C^{*}(G, X)$ type $I$. Then the closure of the ideal $m(A)$ of continuous trace elements of $A$ corresponds, under the bijection of Lemma 16, to the G-invariant open subset $Z=\{x \in X \mid x$ has a wandering compact neighborhood $U$ such that $G U$ is closed in $X\}$.

Proof. First we observe that for any $C^{*}$-algebra $B$, the open subset $\mathcal{O}_{I} \cong I^{\wedge}$ of $B^{\wedge}$ corresponding to the closed ideal $I=I_{B}=m(B)^{-}$ consists precisely of those (equivalence classes of) $\pi$ such that

(i) for some ideal $J$ of $B$ which is a continuous trace algebra, $\pi \in \mathcal{O}_{J}$, and (ii) $\pi$ has a neighborhood basis in $A^{\wedge}$ consisting of closed sets. This is proved as follows: Let $\pi$ be in $\mathscr{O}_{I}$. Then there exists a positive $a \in m(A)$ such that $\pi(a)=1$. The set of $\iota$ such that $\iota(a) \geqq$ $1 / 2$ is a closed neighborhood of $\pi$ contained in $\mathscr{O}_{I}$, and since $\mathscr{O}_{I}$ is Hausdorff and $\pi$ has a neighborhood basis consisting of compact subsets iof $B^{\wedge}$, it has a basis consisting of closed subsets of $\mathcal{O}_{I}$. Hence (ii) holds, and (i) is obvious (take $J=I$ ). Conversely, if $\pi$ satisfies (i) and (ii) let $J_{0}$ be an ideal of $B$ such that $\pi \in \mathcal{O}_{J_{0}}$ and $\mathcal{O}_{J_{0}}^{-} \subseteq \mathcal{O}_{J}$. Then for any positive $\alpha$ in the Pedersen ideal $\kappa\left(J_{0}\right)$ the function $\iota \mapsto \operatorname{Tr} \iota(a)$ is continuous on $\mathcal{O}_{J}$ (since $\kappa\left(J_{0}\right) \cong \kappa(J) \leqq m(J)$ ) and vanishes off $\mathcal{O}_{J_{0}}$, hence is continuous on all of $B^{\wedge}$. Therefore $\kappa\left(J_{0}\right) \subseteq m(B)$, so $J_{0} \subseteq I$ and $\pi \in \mathcal{O}_{1}$.

We apply this result to our algebra $A$, assuming first that $\pi$ is an arbitrary element of $\mathcal{O}_{I_{A}}$. Then Lemma 16 and Theorem 17 imply that any ideal $J$ as above corresponds to an open $G$-invariant 
set $Y$ in which every compact set is wandering. The fact that $\pi$ does not annihilate $J$ implies that the orbit corresponding to $\pi$ lies in $Y$. If $x$ denotes a point in this orbit, then $x$ has a compact wandering neighborhood $N$; taking a closed neighborhood $V$ of $G x$ in $X / G \cong A^{\wedge}$ with $V$ contained in the image of $N$, we see $U=$ $\sigma^{-1}(V) \cap N$ (where $\sigma: X \rightarrow X / G$ is the canonical projection) is a compact wandering neighborhood of $x$ whose saturation under $G$ is closed. It follows that the open set corresponding to $I$ is contained in $Z$.

The reverse inclusion is proved by a similar argument.

Curiously enough, it is possible to deduce a nonobvious fact about transformation groups from the preceding theorem and standard results of $C^{*}$-algebra theory.

Corollary 19. Let $G$ act freely on $X$, with $G$ and $X$ second. countable and with every orbit of $X$ locally closed. Then $X$ contains a point with a wandering neighborhood.

Proof. $C^{*}(G, X)$ is type $I$, by [14]. Since any type I algebra has an ideal which is a continuous trace algebra the corollary follows from Lemma 16 and the theorem above.

In fact the conclusion of the above corollary can be strengthened to say that the set of points with wandering neighborhoods is dense in $X$, since the set of such points is $G$-invariant and so the complement of its closure is a $G$-space satisfying the same hypotheses.

We conclude with an example of a free action of $\boldsymbol{R}$ on a closed subset of $\boldsymbol{R}^{3}$ for which the orbit space is Hausdorff but the associated $C^{*}$-algebra is not a continuous trace algebra.

EXAMPLE. The space $X$ consists of countably many orbits, with the points $x_{0}=(0,0,0)$ and $x_{n}=\left(2^{-2 n}, 0,0\right)(n=1,2, \cdots)$ as a family of representatives for the orbits. We describe $(\boldsymbol{R}, X)$ by giving the $\operatorname{maps} \varphi_{n}: R \rightarrow X \quad s \mapsto s x_{n}$ :

$$
\varphi_{0}(s)=(0, s, 0) \text { for all } s ;
$$

while for $n \geqq 1$,

$$
\varphi_{n}(s)=\left\{\begin{array}{cr}
\left(2^{-2 n}, s, 0\right) & s \leqq n \\
\left(2^{-2 n}-\frac{(s-n)}{\pi} 2^{-2 n-1}, n \cos (s-n), n \sin (s-n)\right) & n<s<n+\pi \\
\left(2^{-2 n-1}, s-\pi-2 n, 0\right) & s \geqq n+\pi,
\end{array}\right.
$$


Thus each orbit consists of two vertical half lines connected by a halfcircle. It can readily be checked that the action we get is jointly continuous, with the orbit space $X / \boldsymbol{R}$ homeomorphic to the subset $\left\{x_{0}, x_{1} \cdots\right\}$ of $\boldsymbol{R}^{3}$, and that $x_{0}$ has no wandering neighborhoods. The $C^{*}$-algebra for this transformation group has as the closure of its set of continuous trace elements the ideal $C^{*}(\boldsymbol{R}, Z)$ where $Z=\cup \boldsymbol{R} x_{n}(n=1,2, \cdots)$. It is an extension of this ideal by a copy of $\mathscr{K}\left(L^{2}(\boldsymbol{R})\right)$, and is thus a "generalized continuous trace algebra" whose composition series is of length two.

We plan to investigate in a future paper the structure of the $C^{*}$-algebras for this and related transformation groups.

\section{REFERENCES}

1. C. A. Akemann, G. K. Pedersen and J. Tomiyama, Multipliers of $C^{*}$-algebras, J. Functional Analysis, 13 (1973), 277-301.

2. L. G. Brown, R. G. Douglas, and P. A. Fillmore, Unitary equivalence modulo the compact operators and extensions of $C^{*}$-algebras, in Proc. Conf. on Operator Theory, Lecture Notes in Math. no. 345, Springer, Berlin, 1973.

3. - Extensions of $C^{*}$-algebras and K-homology, preprint.

4. R. C. Busby, Double centralizers and extensions of $C^{*}$-algebras, Trans. Amer. Math. Soc., 132 (1968), 79-99.

5. J. Cassels and A. Frohlich, Algebraic Number Theory, Thompson Book Company, 1967.

6. D. E. Cohen, Groups of cohomological dimension one, Lecture Notes in Math. no. 245, Springer, Berlin, 1972.

7. J. Dixmier, Traces sur les $C^{*}$-algebres, Ann. Inst. Fourier, 13(1963), 219-262.

8. - Les $C^{*}$-Algebres et Leurs Representations, $2^{*}$ ed., Gauthier-Villars, Paris, 1969.

9. J. Dixmier and A. Douady, Champs continus d'espaces hilbertiens et de $C^{*}$-algebres, Bull. Soc. Math. France, 91 (1963), 227-284.

10. E. G. Effros and F. Hahn, Locally compact transformation groups and $C^{*}$-algebras, Mem. Amer. Math. Soc., no 75 (1967).

11. J. M. G. Fell. The structure of algebras of operator fields, Acta Math., 106 (1961), 233-280.

12. A. M. Gleason, Spaces with a compact Lie group of transformations, Proc. Amer. Math. Soc., 1 (1950), 35-43.

13. J. Glimm, Families of induced representations, Pacific J. Math., 12 (1962), 885-911. 14. E. C. Gootman, The type of some $C^{*}$ and $W^{*}$-algebras associated with transformation groups, Pacific J. of Math., 48 (1973), 93-106.

15. J. Kaminker and C. Schochet, Steenrod homology and operator algebras, Bull. Amer. Math. Soc., 81 (1975), 431-434.

16. C. Lance, On nuclear $C^{*}$-algebras, J. Functional Analysis, 12 (1973), 157-176.

17. D. Milicic, On $C^{*}$-algebras with bounded trace, Glasnik Mat. J., 8 (1973), 7-21.

18. Z. Nitecki, Differentiable dynamics, M.I.T. Press, Cambridge, 1971.

19. G. Pedersen, Measure theory for $C^{*}$-algebras, Math. Scand., 19 (1966), 131-145.

20. C. Pugh and M. Shub, Axiom A actions, Inventiones Math., 29 (1975), 7-38.

21. M. A. Rieffel, On the uniqueness of the Heisenberg commutation relations, Duke Math. J., 39 (1972), 745-752.

22. J. Rosenberg, The $C^{*}$-algebras of some real and p-adic solvable groups, Pacific J. of Math., 65 (1976), 175-192. 
23. S. Sakai, $C^{*}$-Algebras and $W^{*}$-Algebras, Springer-Verlag, Berlin, 1971.

24. N. Steenrod, The Topology of Fiber Bundles, Princeton University Press, 1951.

25. G. Szego, Orthogonal Polynomials, Amer. Math. Soc. Colloq. Pubs. 23, New York, 1939.

26. H. Takai, On a duality for crossed products of $C^{*}$-algebras, J. Functional Analysis, 19 (1975), 25-39.

27. M. Takesaki, Covariant representations of $C^{*}$-algebras and their locally compact automorphism groups, Acta Math., 119 273-302.

28. - On the cross-norm of the direct product of $C^{*}$-algebras. Tôhoku Math. J. (ser. 2), 16 (1964), 111-122.

29. D. Voiculescu, A non-commutative Weyl-von Neumann theorem, preprint.

30. D. N. Z'ep, Structure of the group $C^{*}$-algebra of the group of affine transformations of a straight line, Functional Anal., 9 (1975), 58-60.

Received March 17, 1976 and in revised form April 4, 1977. Partially supported by an NSF Graduate Fellowship. Final preparation of the manuscript was supported by a Grant-in-Aid from the Graduate Division of the University of California, Berkeley.

Columbia University

NEW YoRK, NY 10027 



\title{
PACIFIC JOURNAL OF MATHEMATICS
}

\author{
EDITORS
}

RICHARD ARENS (Managing Editor)

University of California

Los Angeles, California 90024

C. W. CurTis

University of Oregon

Eugene, OR 97403

C. C. MOORE

University of California

Berkeley, CA 94720
J. DUgundJI

Department of Mathematics

University of Southern Californı

Los Angeles, California 90007

R. Finn and J. Milgram

Stanford University

Stanford, California 94305

\section{ASSOCIATE EDITORS}

E. F. BECKENBACH

\section{SUPPORTING INSTITUTIONS}

\author{
UNIVERSITY OF BRITISH COLUMBIA \\ CALIFORNIA INSTITUTE OF TECHNOLOGY \\ UNIVERSITY OF CALIFORNIA \\ MONTANA STATE UNIVERSITY \\ UNIVERSITY OF NEVADA, RENO \\ NEW MEXICO STATE UNIVERSITY \\ OREGON STATE UNIVERSITY \\ UNIVERSITY OF OREGON \\ OSAKA UNIVERSITY
}

UNIVERSITY OF SOUTHERN CALIFORNIA

STANFORD UNIVERSITY

UNIVERSITY OF TOKYO

UNIVERSITY OF UTAH

WASHINGTON STATE UNIVERSITY

UNIVERSITY OF WASHINGTON
AMERICAN MATHEMATICAL SOCIETY
NAVAL WEAPONS CENTER 


\section{Pacific Journal of Mathematics \\ Vol. 72 , No. 1 \\ January, 1977}

Kazuo Anzai and Shiro Ishikawa, On common fixed points for several

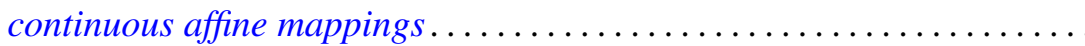

Bruce Alan Barnes, When is a representation of a Banach $*$-algebra

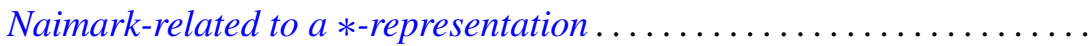

Richard Dowell Byrd, Justin Thomas Lloyd, Franklin D. Pedersen and

James Wilson Stepp, Automorphisms of the semigroup of finite

complexes of a periodic locally cyclic group ...................

Donald S. Coram and Paul Frazier Duvall, Jr., Approximate fibrations and a

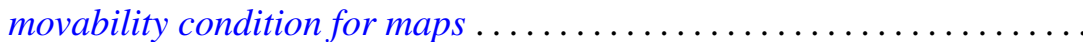

Kenneth R. Davidson and Che-Kao Fong, An operator algebra which is not

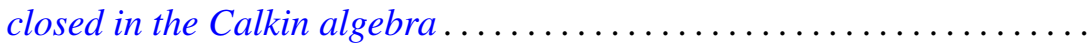

Garret J. Etgen and James Pawlowski, A comparison theorem and oscillation criteria for second order differential systems .............

Philip Palmer Green, $C^{*}$-algebras of transformation groups with smooth

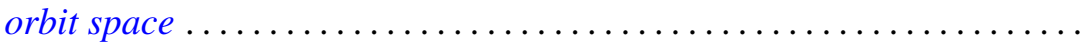

Charles Allen Jones and Charles Dwight Lahr, Weak and norm approximate

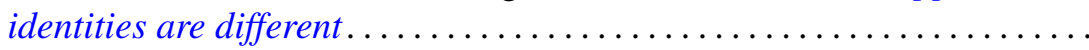

G. K. Kalisch, On integral representations of piecewise holomorphic

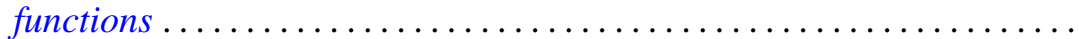

Y. Kodama, On product of shape and a question of Sher

Heinz K. Langer and B. Textorius, On generalized resolvents and

$Q$-functions of symmetric linear relations (subspaces) in Hilbert

space ...................................

Albert Edward Livingston, On the integral means of univalent, meromorphic functions

Wallace Smith Martindale, III and Susan Montgomery, Fixed elements of

Jordan automorphisms of associative rings ..........

R. Kent Nagle, Monotonicity and alternative methods for nonlinear boundary value problems ........................

Richard John O'Malley, Approximately differentiable functions: the $r$ topology.

Mangesh Bhalchandra Rege and Kalathoor Varadarajan, Chain conditions

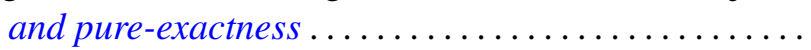

Christine Ann Shannon, The second dual of $C(X)$. .

Sin-ei Takahasi, A characterization for compact central double centralizers of $C^{*}$-algebras .

Theresa Phillips Vaughan, A note on the Jacobi-Perron algorithm. . .

Arthur Anthony Yanushka, A characterization of $\operatorname{PSp}(2 m, q)$ and

$\mathrm{P} \Omega(2 m+1, q)$ as rank 3 permutation groups ......... 